\title{
RESULTS AND GAMES
}

\section{Sixth World Computer Chess Championship}

Ken Thompson

Edmonton, Alberta, Canada

May 28-31, 1989

\begin{tabular}{|c|c|c|c|c|c|c|c|c|c|}
\hline & & rate & perf & 1 & 2 & 3 & 4 & 5 & total \\
\hline 1 & Deep Thought & 1000 & 1418 & $23+\square$ & $16+\square$ & $6+\square$ & $3+\square$ & $4+\square$ & 5.0 \\
\hline 2 & Bebe & 1000 & 1190 & $20+\square$ & $3-\square$ & $18+\square$ & $16+\square$ & $7+\square$ & 4.0 \\
\hline 3 & Cray Blitz & 1000 & 1120 & $15+\square$ & $2+\square$ & $4=\square$ & $1-\square$ & $11+\square$ & 3.5 \\
\hline 4 & Hitech & 1000 & 1120 & $11+\square$ & $13+\square$ & $3=\square$ & $6+\square$ & $1-\square$ & 3.5 \\
\hline 5 & Mephisto X & 1000 & 1120 & $18-\square$ & $17+\square$ & $8+\square$ & $10=\square$ & $12+\square$ & 3.5 \\
\hline 6 & Fidelity X & 1000 & 1059 & $21+\square$ & $7+\square$ & $1-\square$ & $4-a$ & $10+\square$ & 3.0 \\
\hline 7 & Merlin & 1000 & 1059 & $24+\square$ & $6-\square$ & $19+\square$ & $9+\square$ & $2-\square$ & 3.0 \\
\hline 8 & AI Chess & 1000 & 1059 & $22+\square$ & $9=\square$ & $5-\square$ & $12=\square$ & $16+\square$ & 3.0 \\
\hline 9 & Sun Phoenix & 1000 & 999 & $12+\square$ & $8=\square$ & $10=\square$ & $7-\square$ & $14=\square$ & 2.5 \\
\hline 10 & Novag X & 1000 & 999 & $17=\square$ & $18+\square$ & $9=\square$ & $5=\square$ & $6-\square$ & 2.5 \\
\hline 11 & Much & 1000 & 999 & $4-\square$ & $14=\square$ & $15+\square$ & $21+\square$ & $3-\bar{\square}$ & 2.5 \\
\hline 12 & Zarkov & 1000 & 999 & $9-\square$ & $22+\square$ & $13+\square$ & $8=\square$ & $5-\square$ & 2.5 \\
\hline 13 & Quest X & 1000 & 999 & $14+\square$ & $4-\square$ & $12-\square$ & $22+\square$ & $20=\square$ & 2.5 \\
\hline 14 & Y!89 & 1000 & 999 & $13-\square$ & $11=\square$ & $17=\square$ & $18+\square$ & $9=\square$ & 2.5 \\
\hline 15 & $\mathrm{BP}$ & 1000 & 999 & $3-\square$ & $20+\square$ & $11-\square$ & $19=\square$ & $23+\square$ & 2.5 \\
\hline 16 & Rebel X & 1000 & 940 & $19+\square$ & $1-\square$ & $21+\square$ & $2-\square$ & $8-\square$ & 2.0 \\
\hline 17 & Kallisto & 1000 & 940 & $10=\square$ & $5-\square$ & $14=\square$ & $20=\square$ & $19=\square$ & 2.0 \\
\hline 18 & Waycool & 1000 & 940 & $5+\square$ & $10-\square$ & $2-\square$ & $14-\square$ & $24+\square$ & 2.0 \\
\hline 19 & $\operatorname{Rex}$ & 1000 & 940 & $16-\square$ & $23+\square$ & $7-\square$ & $15=\square$ & $17=\square$ & 2.0 \\
\hline 20 & Pandix & 1000 & 940 & $2-\square$ & $15-\square$ & $23+\square$ & $17=\square$ & $13=\square$ & 2.0 \\
\hline 21 & Dappet & 1000 & 940 & $6-\square$ & $24+\square$ & $16-\square$ & $11-\square$ & $22+\square$ & 2.0 \\
\hline 22 & Shess & 1000 & 808 & $8-\square$ & $12-\square$ & $24+\square$ & $13-\square$ & $21-\square$ & 1.0 \\
\hline 23 & Moby & 1000 & 808 & $1-\square$ & $19-\square$ & $20-\square$ & $24+\square$ & $15-\square$ & 1.0 \\
\hline 24 & Centaur & 1000 & 581 & $7-\square$ & $21-\square$ & $22-\square$ & $23-\square$ & $18-\square$ & 0.0 \\
\hline
\end{tabular}

\section{Round 1}

\section{Moby - Deep Thought \\ D01/15 4 Qd3}

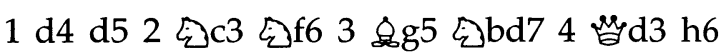
5 \&h4 c6 6 乞f3 g6 7 h3 \&g7 8 g4 ka5 9 a3

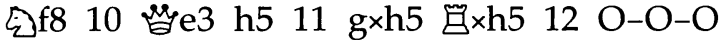

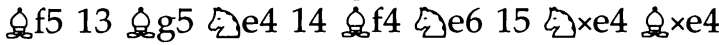

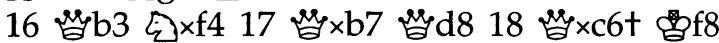

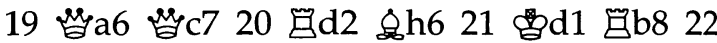

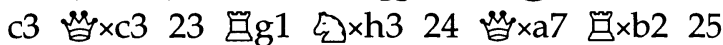
ㅂaga5 自b1\# 0-1

\section{Cray Blitz - BP C24/05 5 e:d5}

1 e4 e5 2 \&c4 £f6 3 d3 c6 4 ¿f3 d5 5 exd5 cxd5 6 \&b3 \&b4t 7 c3 \&d6 8 \&g5 \&e6 9

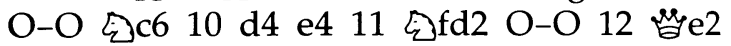

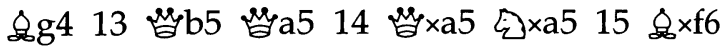
¿ $\times$ b3 16 axb3 gxf6 17 c4 f5 18 cxd5 \&f4 19

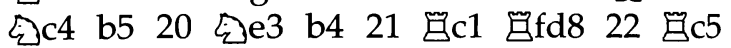

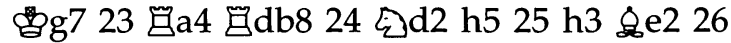

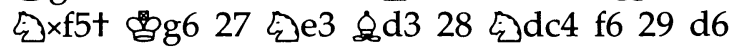

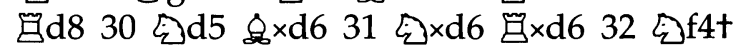

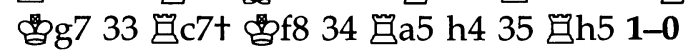

\section{Much - Hitech \\ D02/04 3. Bf5}

1 乞f3 d5 2 g3 £c6 3 d4 \&f5 4 \&g2

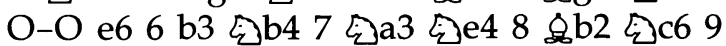

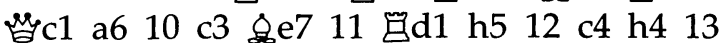

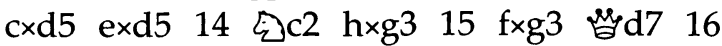

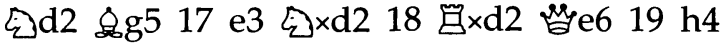

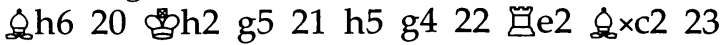

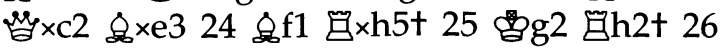

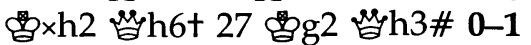

\section{Fidelity $X$ - Dappet B80/11 17 g5}

1 e4 c5 2 ¿f3 d6 3 d4 cxd4 4 乡xd4 乡f6 5

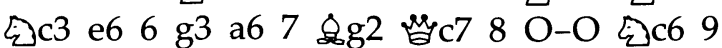

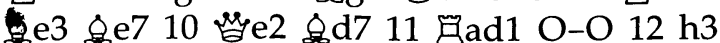
b5 13 a3 $气 \times d 414$ \&xd4 \&c6 15 g4 自fe8 16 
f4 쌈b7 17 g5 $气 d 718$ h4 e5 19 fxe5 dxe5 20 \&e3 a5 21 쌈f 2 gf8 22 气d5 Gae8 $23 \mathrm{~h} 5 \mathrm{a} 4$

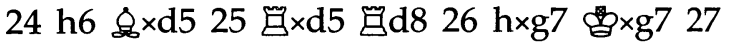

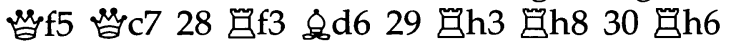

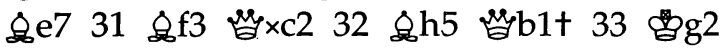

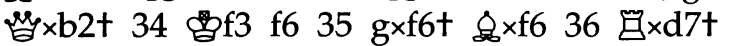
g $\times$ d7 37 쌈 $\times d 7+1-0$

\section{Waycool - Mephisto X \\ B07/05 3. c6}

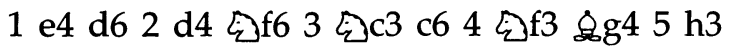
\&h5 6 \&d3 e6 7 gg1 \&e7 8 g4 \&g6 9 e5

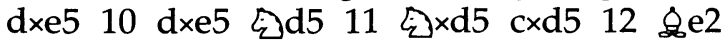

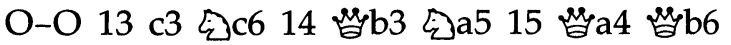

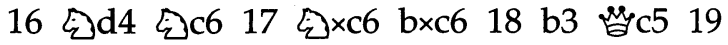

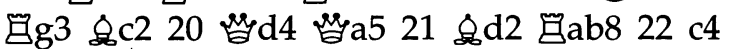

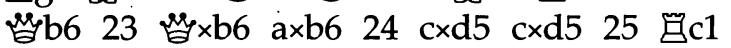
\&e4 26 Ic7 \&d8 27 Ga7 \&h4 28 Ic3 而a8 29. $\Xi \times a 8 \Xi \times a 830$ a4 h6 31 \&e3 $\Xi b 832$ fi \&g5 33 f4 \&d8 34 \&b5 g5 35 ffy gxf4 36 \&.f4 \&g5 37 \&e3 f6 38 exf6 \&xe3+ 39 \&

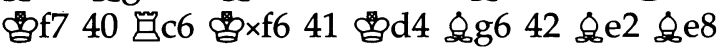

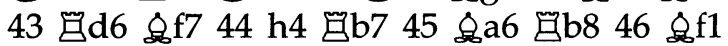
Eb7 $47 \quad \& g 2$ Eb8 48 \&f3 \&g8 49 \&h1 \&f7 50 \&g2 51 be5 b5 52 a5 b4 53 a6 Gb5

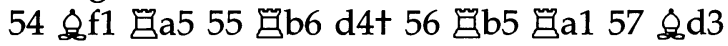

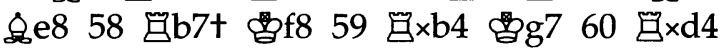
南5+ 61 d $1-0$

\section{Sun Phoenix - Zarkov A18/05 $7 \mathrm{~d} 3$}

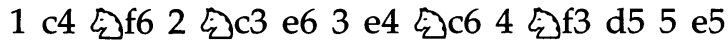

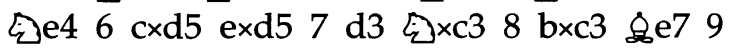
\&e2 O-O 10 O-O f6 $11 \mathrm{~d} 4$ fxe5 $12 \mathrm{~d} \times \mathrm{e} 5$ \&f5 13 \&e3 a6 14 c4 \&e4 15 cxd5 \& $\&$ d5 16 빰 1

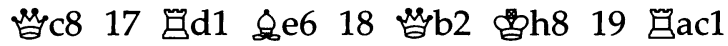
\&g4 20 a3 h6 21 h3 \&e6 22 今d2 留d8 23

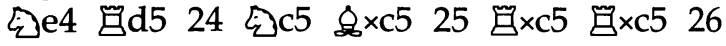

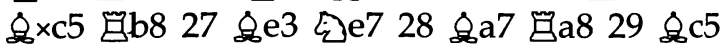

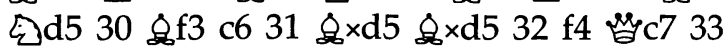

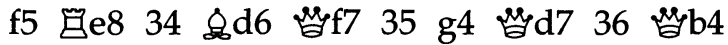

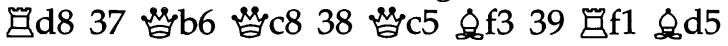

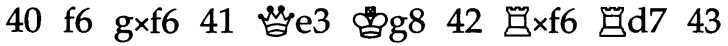
찹 $\times$ h6 1-0

\section{Pandix - Bebe A06/04 3. Bf5}

$1 £ f 3$ c5 2 b3 d5 3 \&b2 \&f5 4 e3 e6 5 \&b5t

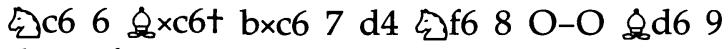

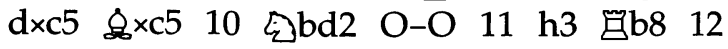

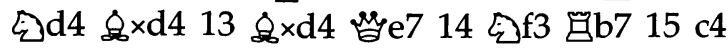

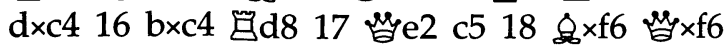

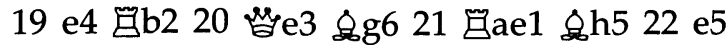

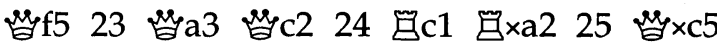

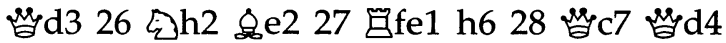

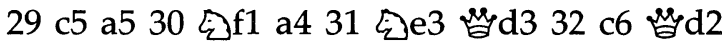

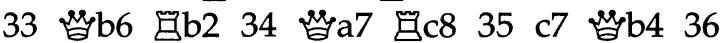

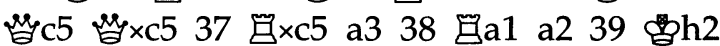
\&d3 40 g g 3 h7 $41 \mathrm{f} 4$ \&e4 $42 \mathrm{~h} 4$ 畐d2 43

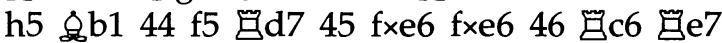

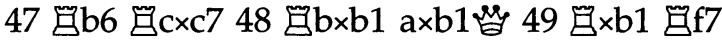

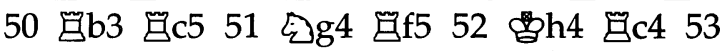

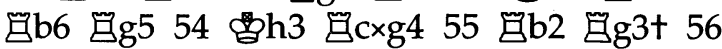

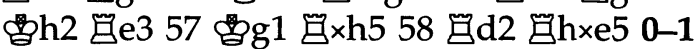

$$
\begin{aligned}
& \text { Rebel X-Rex } \\
& \text { D25/23 12. B:e2 }
\end{aligned}
$$

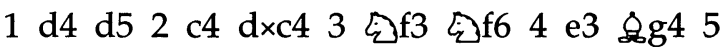

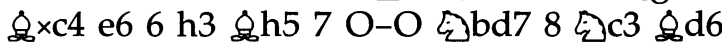

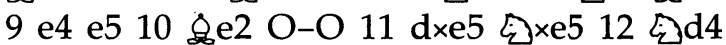

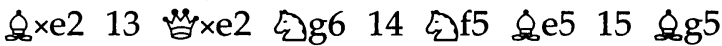

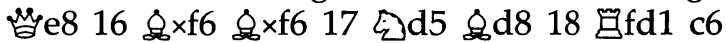

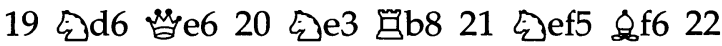

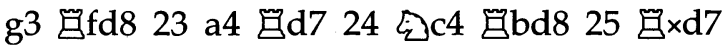

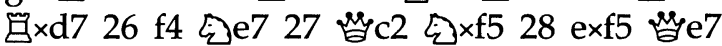

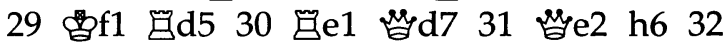

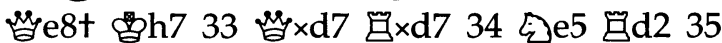

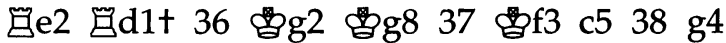

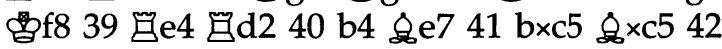

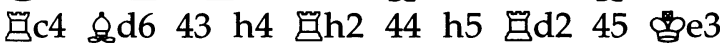

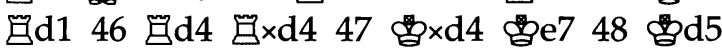
\&b4 49 g5 f6 50 g×h6 g×h6 51 §g4 \&d2 52

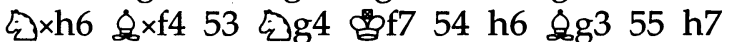

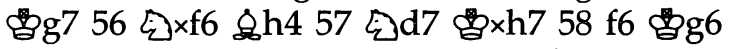

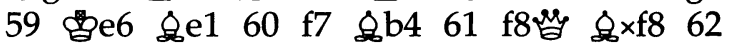

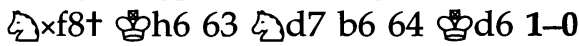

$$
\begin{gathered}
\text { Kallisto - Novag } X \\
\text { C80/02 } 11 \text { Nc3 }
\end{gathered}
$$

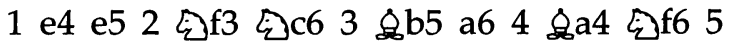

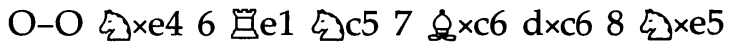
\&e7 $9 \mathrm{~d} 4$ ¿e6 10 \&e3 O-O 11 ¿c3 \&f6 12

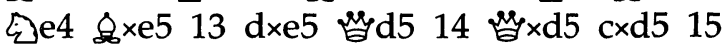

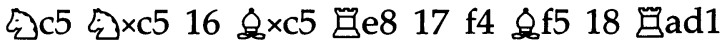

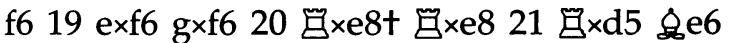
22 月d3 b6 23 \&d4 24 a4 \&f5 25 月ac3 c5 $26 \&$ e3 \& $\times$ 22 $27 \& \times$ \&5 \& $\& 428 \& \times b 6$ घb8 29

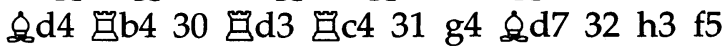
33 g5 \&b5 34 目d2 35 g h.g5 37 fxg5 f4 38 ff3 39 \&c3 \&c6t 40

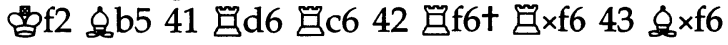
\&e8 $\frac{1 / 2-1 / 2}{2}$

$$
\begin{gathered}
\text { Quest X - Y!89 } \\
\text { A09/15 } 4 \text { Qa4 }
\end{gathered}
$$

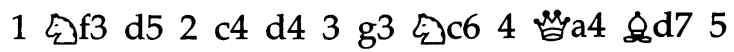




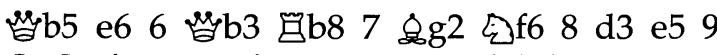
$\mathrm{O}-\mathrm{O} \& \mathrm{e} 710$ \&5 $\mathrm{O}-\mathrm{O} 11$ ¿bd2

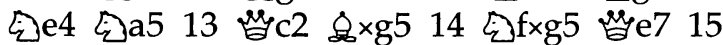

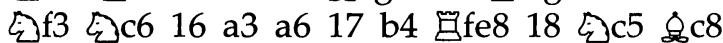

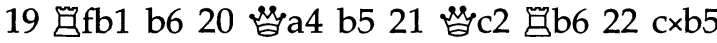

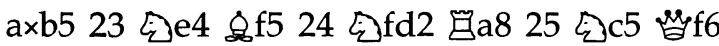
26 a4 \&c8 27 ¿de4 쌈f5 28 a5 abb8 29 h3

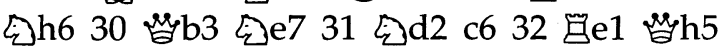

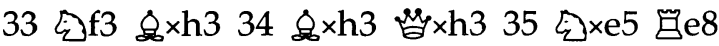

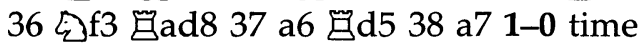

\section{Centaur - Merlin \\ C42/19 13 B:d5}

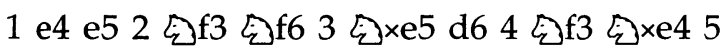
d4 d5 6 \&d3 \&e7 7 O-O $8 \mathrm{c} 68$ Ge1 \&g4 9

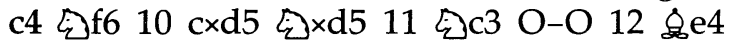

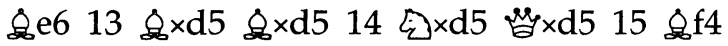

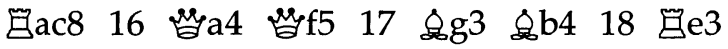
Gfd8 19 a3 \&e7 20 gd1 \&f6 21 h3 b5 22

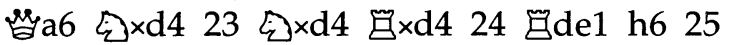

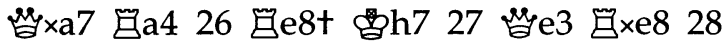

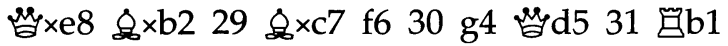

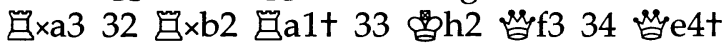
0-1

\section{AI Chess - Shess \\ E09/03 9. Ba6}

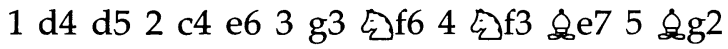

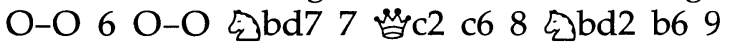

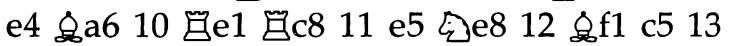

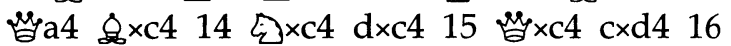

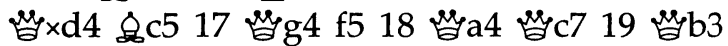

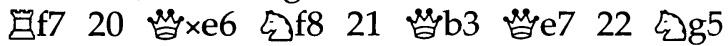

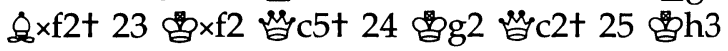

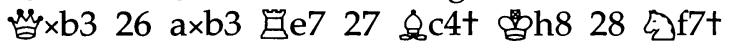

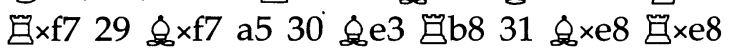

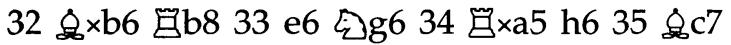

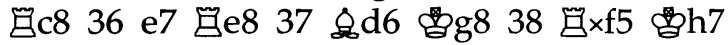

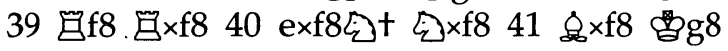

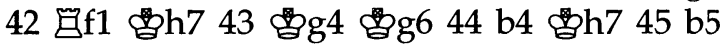

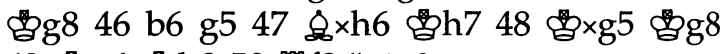
49 gुg6 50 管f 8 \# 1-0

\section{Round 2}

\section{Deep Thought - Rebel X B17/05 11. a6}

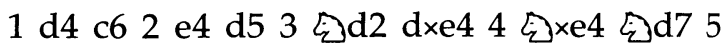

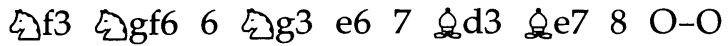

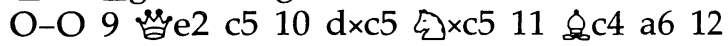

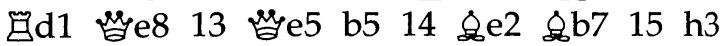

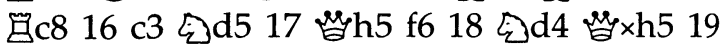

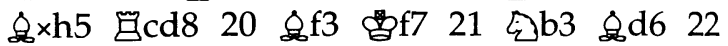

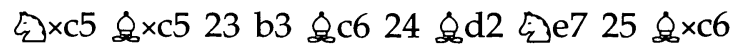

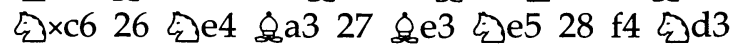

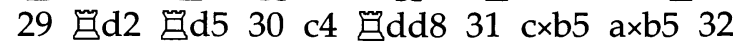

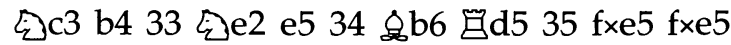

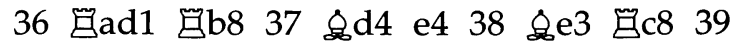

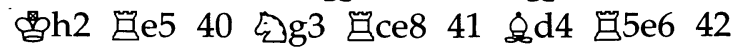

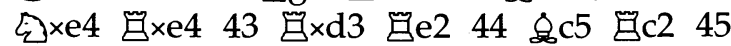

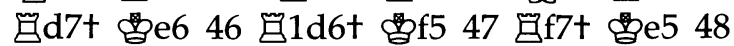

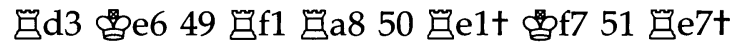
g 52 gूg3+ 1-0

$$
\begin{gathered}
\text { Bebe - Cray Blitz } \\
\text { B84/09 } 14 \text { Qf3 }
\end{gathered}
$$

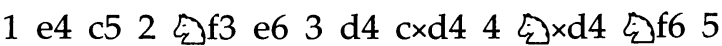
今c3 d6 6 \&e2 a6 7 O-O 쌈7 8 f4 b5 9 \&f3

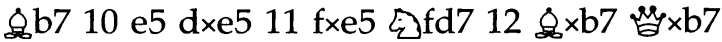

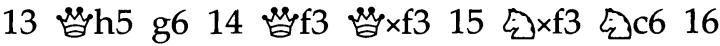

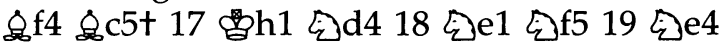

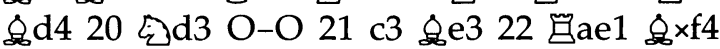

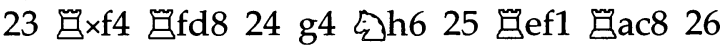

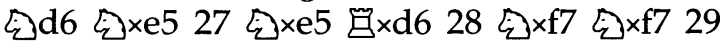

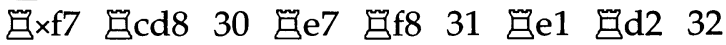

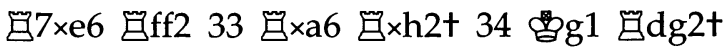

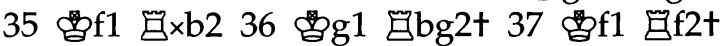

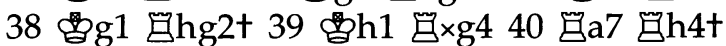

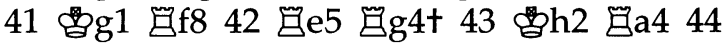

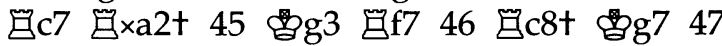

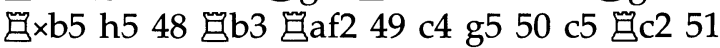

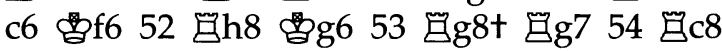

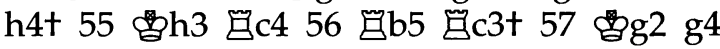
58 gb2 h3+ 59 ghh2 60 gb1 g3+ 61

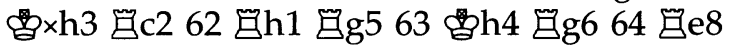

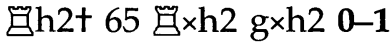

$$
\begin{gathered}
\text { Hitech - Quest X } \\
\text { C42/10 8. h6 }
\end{gathered}
$$

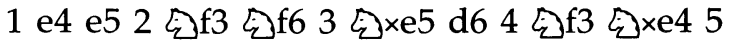

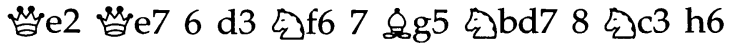
9 \&e3 $气$ e5 10 气xe5 dxe5 11 d4 exd4 12 $8 \times d 4$ c6 $13 \quad \& \times f 6 \quad \mathrm{~g} \times \mathrm{f6} \quad 14$ O-O-O \&e6 15

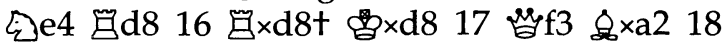

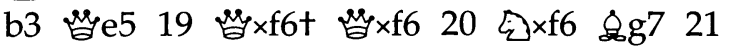

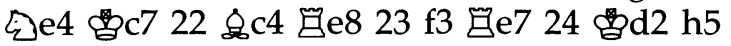

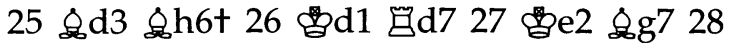

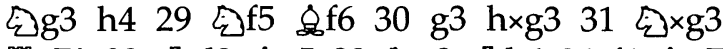

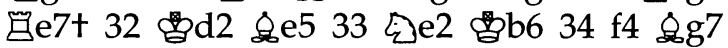

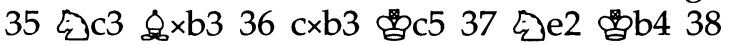

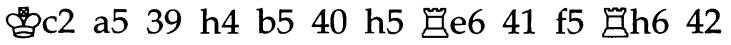
今c3 \&f6 43 \&e2 a4 44 b×a4 b×a4 45 留b1十 क्व $1-0$

$$
\text { Merlin - Fidelity } X
$$
B01/18 9 Bg2 


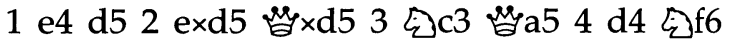
5 ¿f3 \&g4 6 h3 \&h5 7 g4 \&g6 8 ¿f e6 9

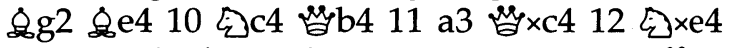

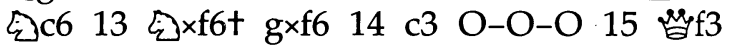

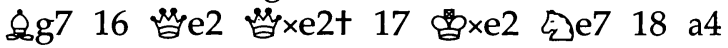
क् \&e4 h6 $23 \quad \& b 7$ e5 24 \&e3 exd4 $25 \quad \& \times d 4$ לg6 26 Gad1 乡f4t 27 gie3 28 e6

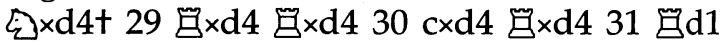
Exd1 32 ×d1 f5 33 c2 f×g4 $34 \mathrm{~h} \times \mathrm{g} 4 \mathrm{c5} 35$

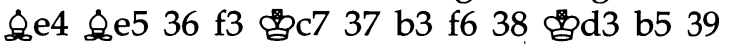

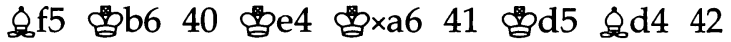
\&e6 ta5 43 ge4 44 by 445 bxc4 b×c4 46 te4 \&e5 47 \&d7 c3 48 कd 3 b3 $49 \& c 6$ a5 50 \&b5 c2 0-1

\section{AI Chess - Sun Phoenix A81/07 5 Bf4}

$1 \mathrm{~d} 4 \mathrm{f} 52 \mathrm{~g} 3$ 乞f6 3 \&g2 e6 4 乞f3 \&e7 5 \&f4 O-O 6 O-O $5 \mathrm{c} 6 \quad 7$ ¿c3 d6 8 d5 exd5 9

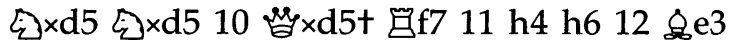

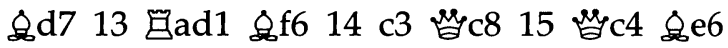

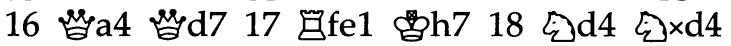

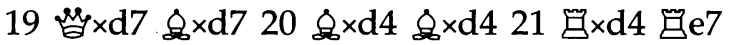
22 घb4 \&c6 23 \& $\times c 6$ b×c6 24 घb7 gुg8 25

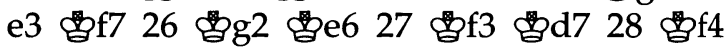
c5 29 日d1

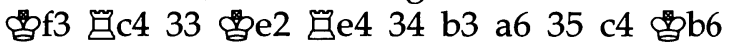
36 h5 g5 37 gd5 f4 38 g×f4 g×f4 39 gf5 f×e3

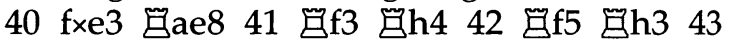

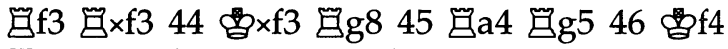

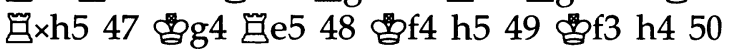

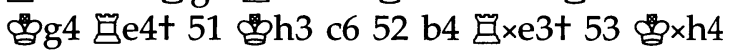

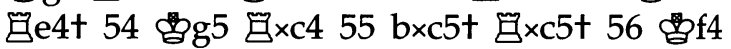
Gh5 57 Gb4t 58 gb3 c5 59 घa3

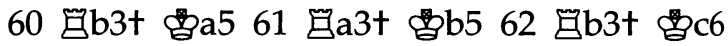

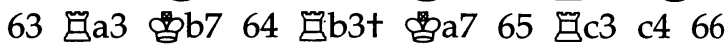

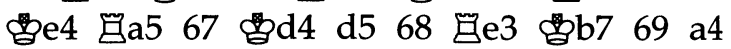

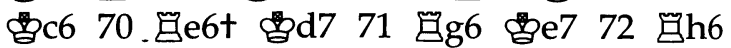
ff8 73 日h7 c3 74 目c7 1/2-1/2

\section{Novag $\mathbf{X}$ - Waycool B33/28 12. Be6}

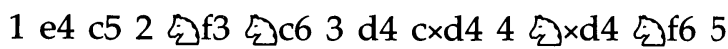

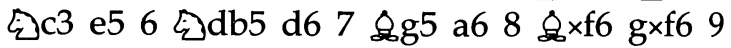
§a3 b5 10 £d5 f5 11 exf5 \&xf5 12 c3 \&e6

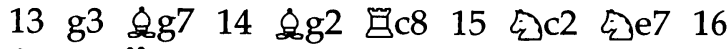

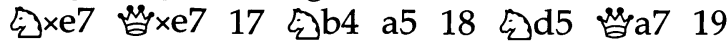
쌉e2 참5 20 O-O-O b4 21 bुb1 \&f5t 22

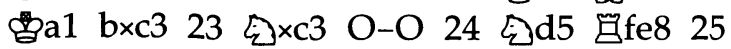

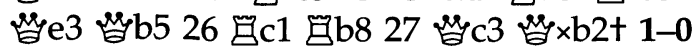

\author{
Mephisto X - Kallisto \\ D06/13 3. d:c4
}

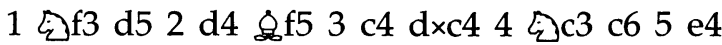

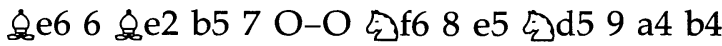
$10 \fallingdotseq \mathrm{b} 1 \quad \mathrm{c} 711 \quad \xi \mathrm{bd} 2 \mathrm{c} 312 \mathrm{~b} \times \mathrm{c} 3 \mathrm{~b} \times \mathrm{c} 313$

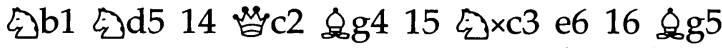

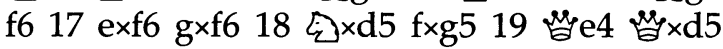

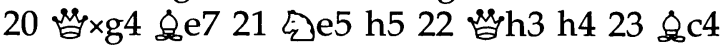

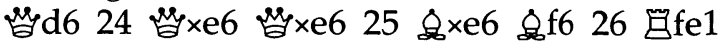

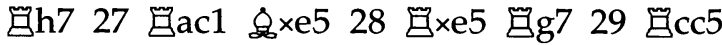

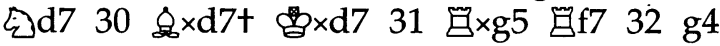

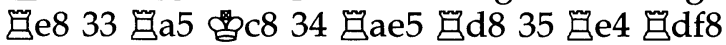

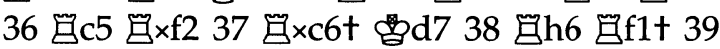
gign $28 \mathrm{f} 2+40$ th 1-0 time

\section{Dappet - Centaur B01/13 4. Nc6}

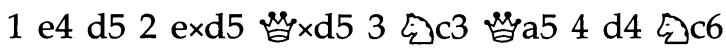

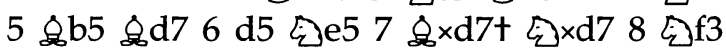

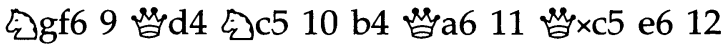

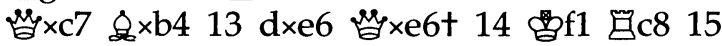

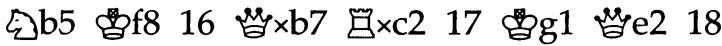

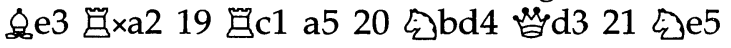
침6 22 Gc8t 1-0

$$
\begin{aligned}
& \text { Y!89 - Much } \\
& \text { B09/31 } 10 \text { h3 }
\end{aligned}
$$

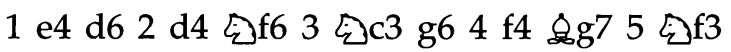
O-O 6 \&d3 2 c6 7 \&e3 e5 8 fxe5 dxe5 9 d5

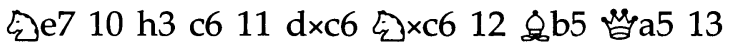
\&×c6 b×c6 14 O-O \&a6 15 छe1 Gad8 16

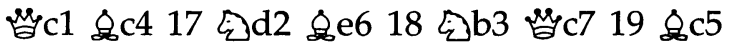

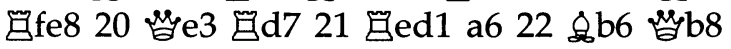

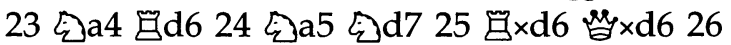

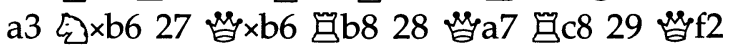

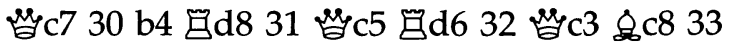

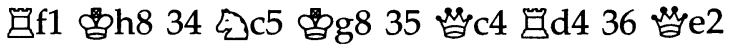

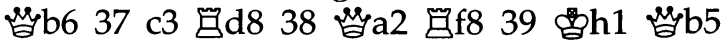

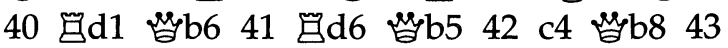

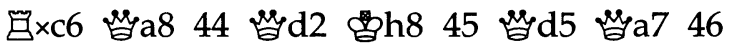

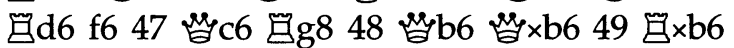

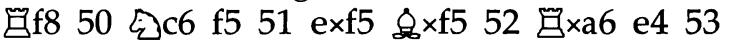

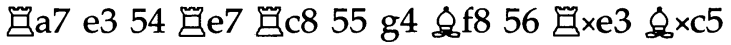

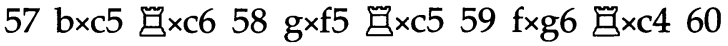

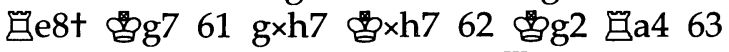

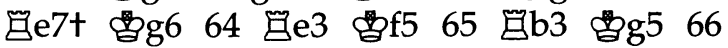

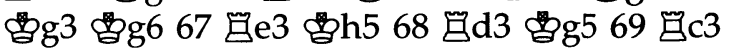

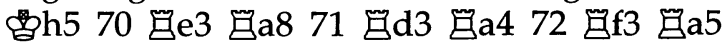

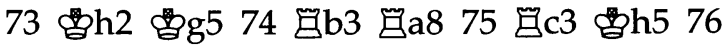

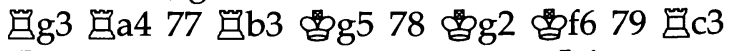

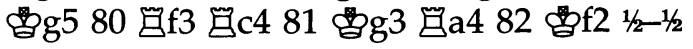




\section{BP - Pandix}

C30/01 2. f5

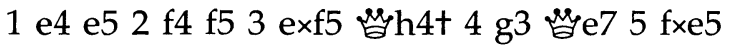

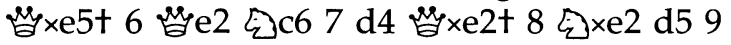
\&h3 \&d6 10 ¿bc3 乡ge7 11 \&g5

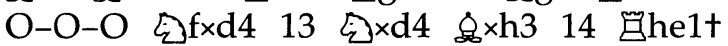

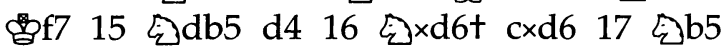

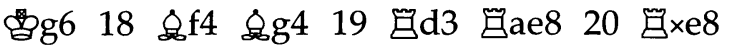

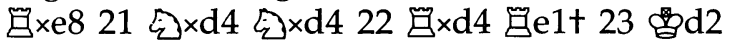

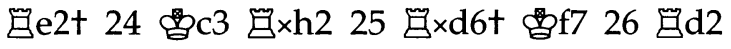
m×d2 $27 \quad$ bed2 b5 28 b3 h5 29 c4 b×c4 30 bxc4 \&e6 31 did3 g6 32 a4 \&d7 33 a5 a6 34 13d4 35 \&c7 g5 36 \&d8t 37 c5

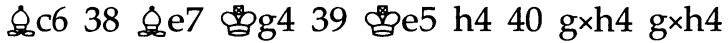

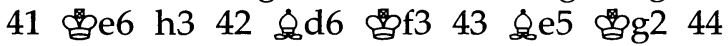
\%d6 \& 45 c6 h2 46 \& 8 h2

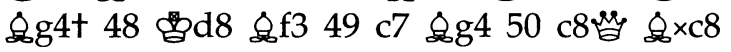

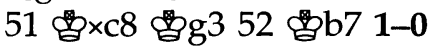

\section{Zarkov - Shess E00/05 6. Nbd7}

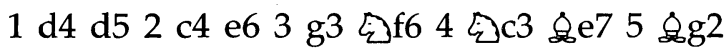

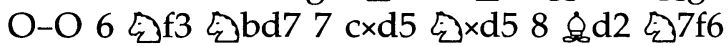

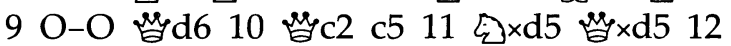

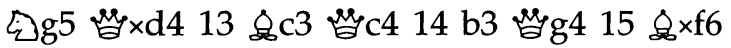

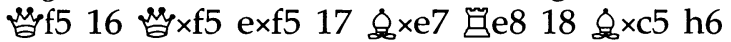

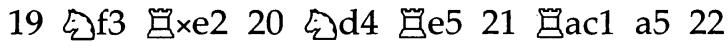

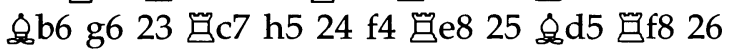

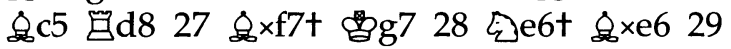
\&xe6t 30 ge1 g5 31 \&c4 冒a6 32 \&×a6

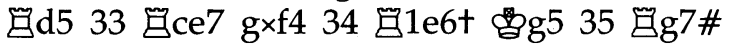
1-0

$$
\begin{aligned}
& \text { Rex - Moby } \\
& \text { B05/17 9. d:e5 }
\end{aligned}
$$

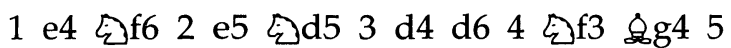
\&e2 e6.6 O-O \&e7 7 c4 §b6 8 ¿c3 O-O 9

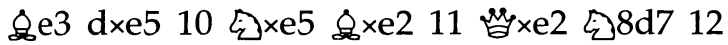

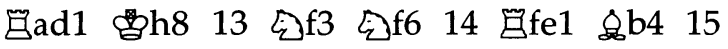
\&d2 참7 16 a3 \&a5 17 b4

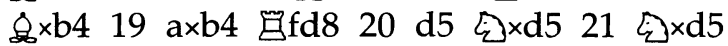

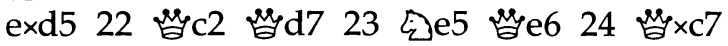

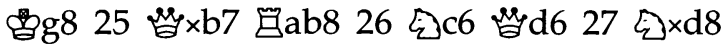

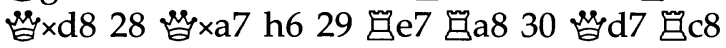

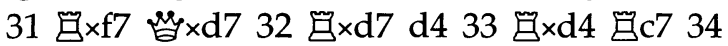

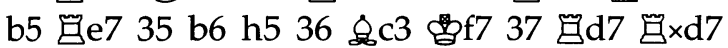
38 日xd7+ $1-0$

\section{Round 3}

Fidelity X - Deep Thought

B05/23 14. f5

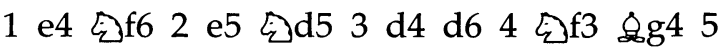
\&e2 e6 6 O-O \&e7 7 c4 $₫ \mathrm{~b} 68 \mathrm{~h} 3$ \&h5 9

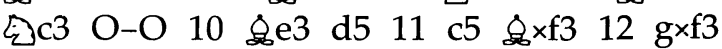

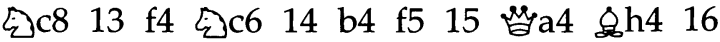

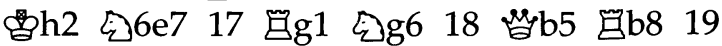

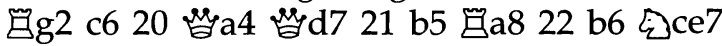

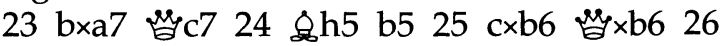

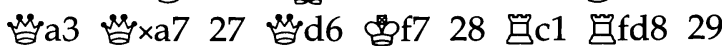
\& $\times g 6+\mathrm{h} \times \mathrm{g} 630$ 쌉b4 冒db8 31 쌉d6 쌉a5 32

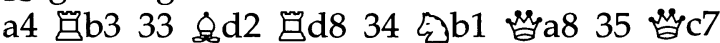

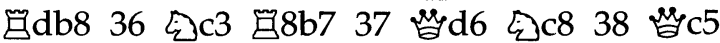

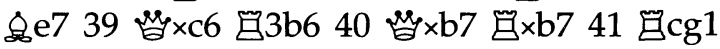
\&f8 $42 \quad \Xi \times g 6 \quad$ घb2 $43 \quad$ \&e1 kㅏㅂa7 $44 \quad$ घ6g3

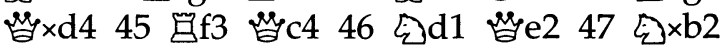

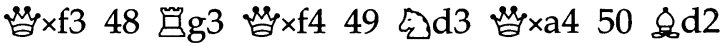

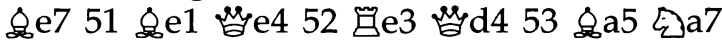

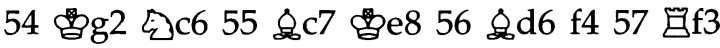
g5 58 ¿c1 \&xd6 59 exd6

$$
\begin{gathered}
\text { Cray Blitz - Hitech } \\
\text { C69/06 } 11 \text { Be3 }
\end{gathered}
$$

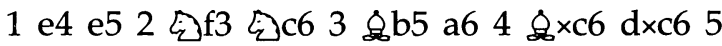

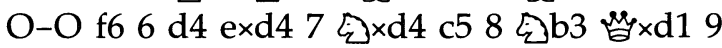
gxd1 \&g4 10 f3 \&e6 11 \&e3 b6 12 a4 \&d6

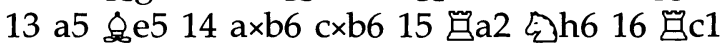

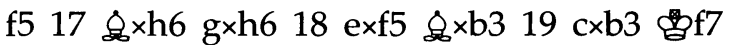

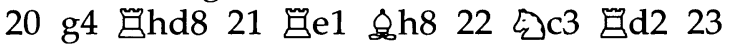

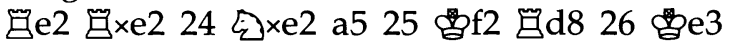

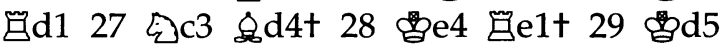

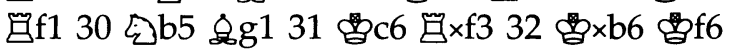

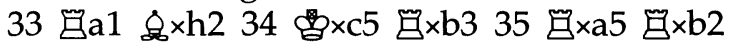

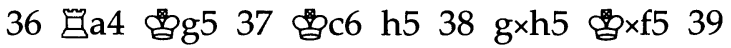

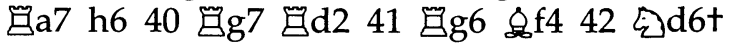

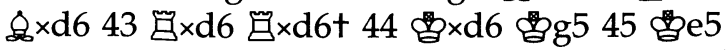

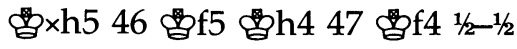

$$
\begin{gathered}
\text { Mephisto X - AI Chess } \\
\text { D70/02 4. Q:d5 }
\end{gathered}
$$

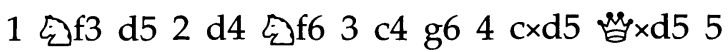

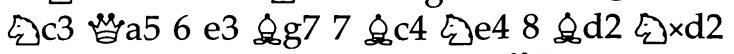

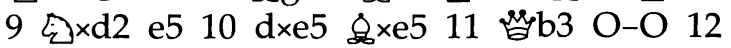

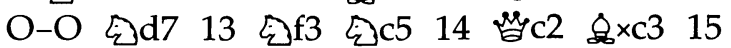

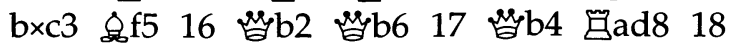

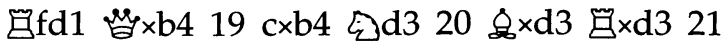

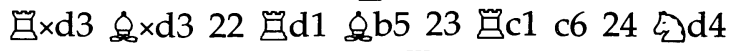
\&d3 25 घc3 \&b1 26 a4 月d8 27 a5 a6 28 h4 f5 29 f3 \&a2 30 gf 2 ga 31 gig3 32

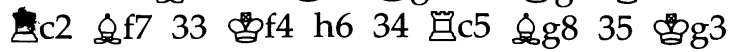




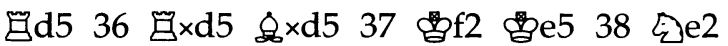
\&c4 39 今c3 \&d3 $40 \mathrm{~g} 4 \mathrm{f} \times \mathrm{g} 441 \mathrm{f} \times \mathrm{g} 4$ \&c2 42

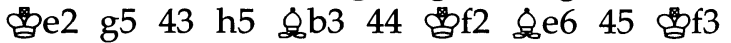

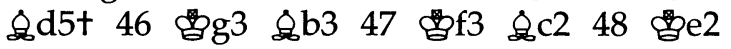

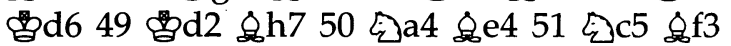

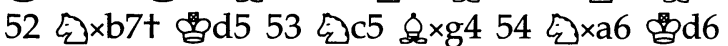
55 今c5 56 e4 $8 \times h 557$ e5 g4 58 e6 g3 59 ge3 \& 60 e7 \&h5 61 ¿e4 \&g6 62 乞 $\times g 3$ c5 63 bxc5 1-0

\section{Sun Phoenix - Novag $X$ A36/19 12. e5}

1 c4 c5 2 ¿c3 $气$ c6 3 g3 g6 4 \&g2 \&g7 5 e4 ¿6 6 ¿ge2 O-O 7 O-O d6 8 d3 §e8 9 h3

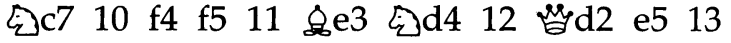

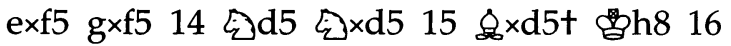

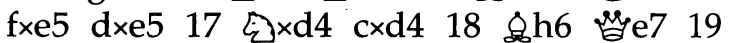

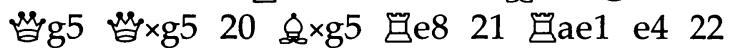
dxe4 fxe4 23 Gxe4 \&xh3 24 Gf7 而xe4 25

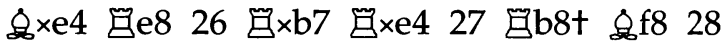

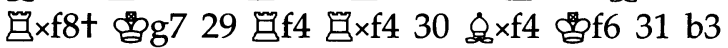

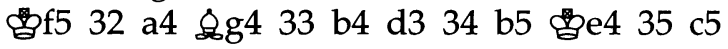

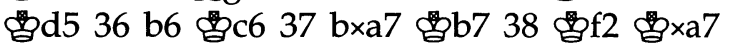

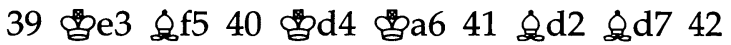

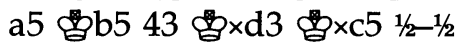

\section{Waycool - Bebe B22/02 4 Bd3}

1 e4 c5 2 c3 d6 3 d4 2 f6 4 \&d3 cxd4 5 cxd4

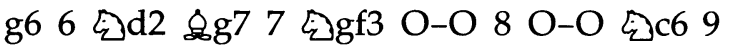

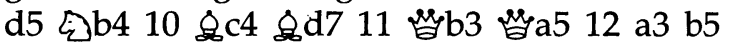

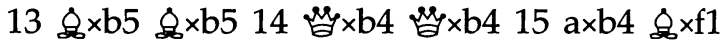
16 百fb8 0-1 time

\section{Rebel X - Dappet D25/17 7 O-O}

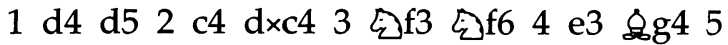
\& 8 c4 e6 6 h3 \&h5 7 O-O c6 8 b3 b5 9 \&e2 \&e7 10 \&b2 O-O 11 ¿e5 \& \&e2 12 찹 $\times$ e2

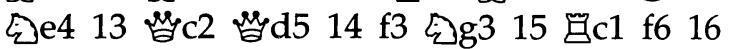

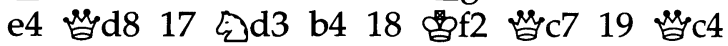

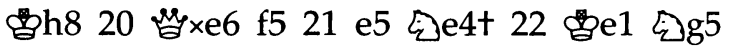

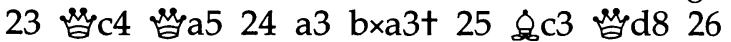

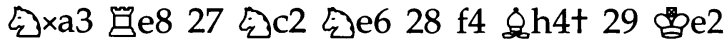

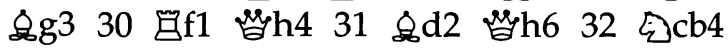

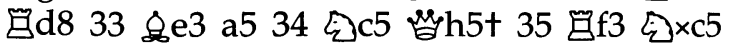

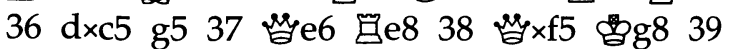

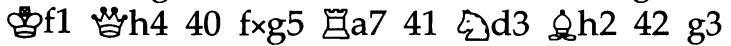

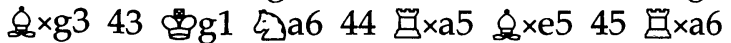

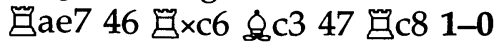

\section{Quest X - Zarkov \\ B30/04 5 Nc3}

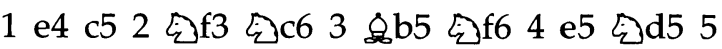

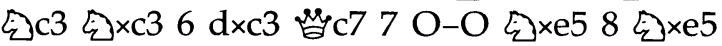

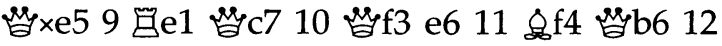
c4 a6 13 쌉a3 쌈a7 14 \&a4 b5 15 cxb5 axb5

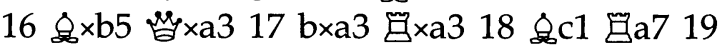
a4 \&e7 20 \&4 O-O $21 \& b 8$ mb7 22 \&e5 d5 23 h3 c4 24 \&c6 五b6 25 \&b5 \&c5 26 \&c3 Øb7 27 \&c6 Ic7 28 \&b5 घa7 29 a5 \&a6 30

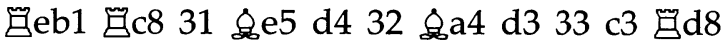
34 \&c6 d2 35 \&f4 e5 36 \& $\times 5$ 包e7 37 \&g3

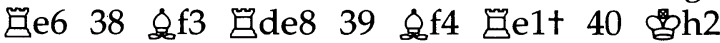

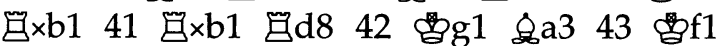

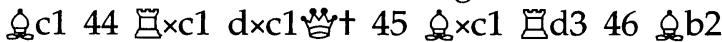

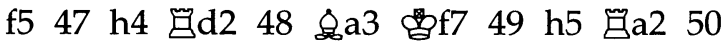
\&b4 $51 \mathrm{~h} 6 \mathrm{~g} \times \mathrm{h} 652 \mathrm{~g}$ ( Ga1t 53 th2

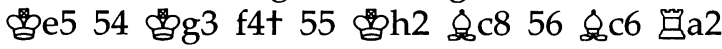

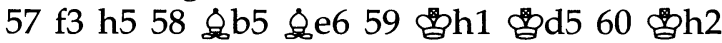

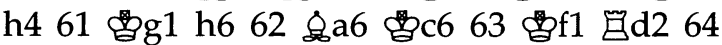

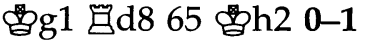

$$
\begin{aligned}
& \text { Rex - Merlin } \\
& \text { C42/08 7. Bg4 }
\end{aligned}
$$

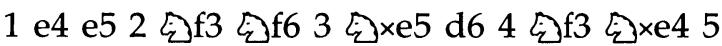

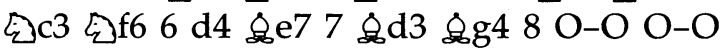

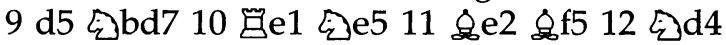

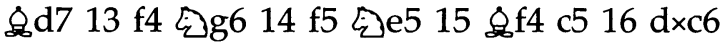

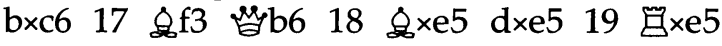
\&d6 20 घe3 c5 21 ¿e6 f×e6 22 \&×a8 c4 23

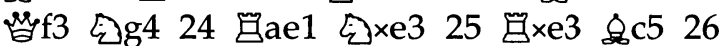

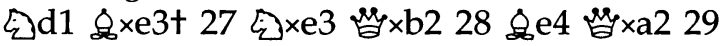

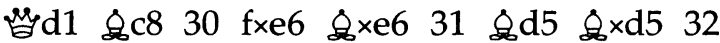

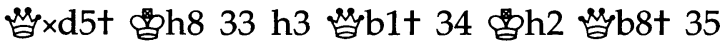

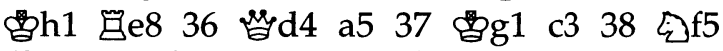

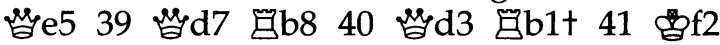

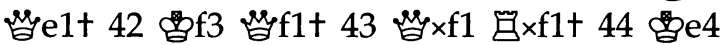
可×55 45 d4 0-1

$$
\begin{aligned}
& \text { Much - BP } \\
& \text { A08/03 } 4 \text { d4 }
\end{aligned}
$$

$1 乞 \mathrm{f} 3 \mathrm{~d} 52 \mathrm{~g} 3 \mathrm{c} 53$ \&g2 $\mathrm{gc} 64 \mathrm{~d} 4 \mathrm{c} 45 \mathrm{O}-\mathrm{O}$

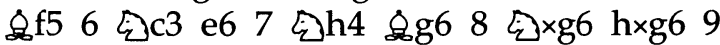
Ie1 \&b4 10 쌈d2

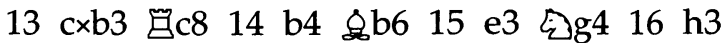

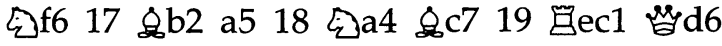

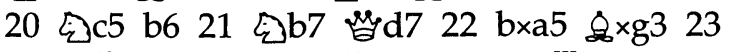

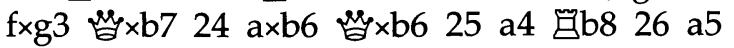

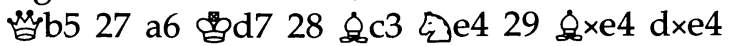

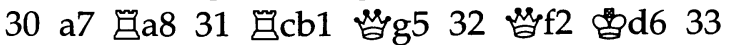
Ga6 쌈f5 34 침b2 g5 35 쌈b7 e5 36 \&b4t 1-0 


$$
\begin{gathered}
\text { Kallisto - Y!89 } \\
\text { B40/04 } 6 \text { Be3 }
\end{gathered}
$$

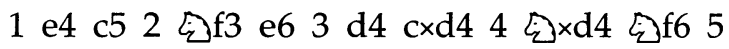

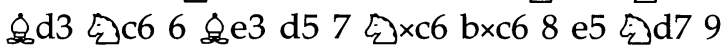

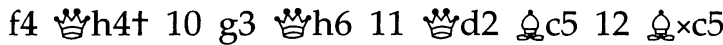

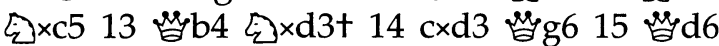

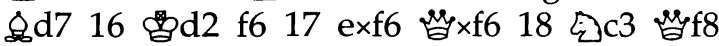

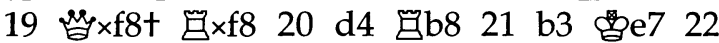

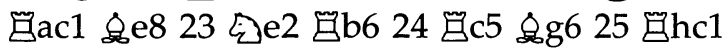

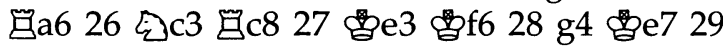

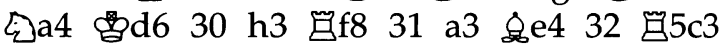

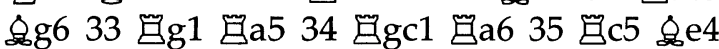

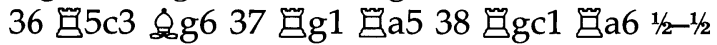

\section{Moby - Pandix D54/03 $10 \mathrm{~g} 3$}

$1 \mathrm{~d} 4$ ¿f6 2 c4 e6 3 ¿c3 d5 4 \&g5 \&e7 5 e3

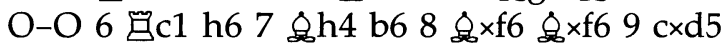
exd5 10 g3 c6 11 \&h3 \&d7 12 \& $\quad$ \&d7 $\quad$ \& $\times d 7$

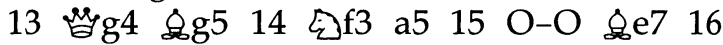

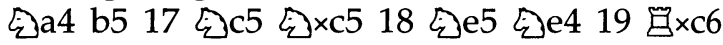

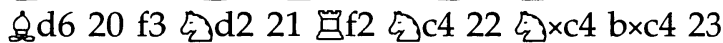
쌉f5 \&b4 $24 \mathrm{a} 3$ \&e7 25 Ije2 $\boxminus \mathrm{e} 826 \mathrm{e} 4 \mathrm{~d} \times \mathrm{e} 4$ $27 \boxminus \times e 4 \Pi b 828 \Xi g 4 \pi \times b 229 \Xi \times c 4 \quad \& \times a 330$

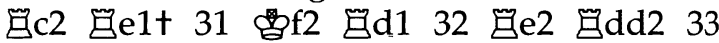

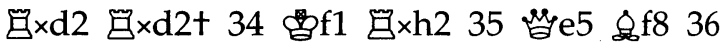

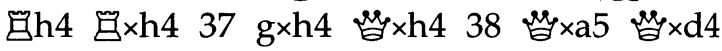
0-1

\section{Shess - Centaur \\ B01/06 4. Nb4}

1 e4 d5 2 exd5 $\leftrightharpoons$ f6 3 d4 4 xd5 4 c4 $\leftrightharpoons$ b4 5 찹a4t

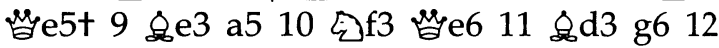

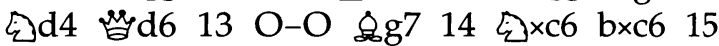

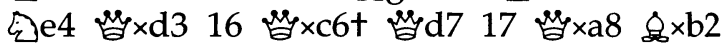

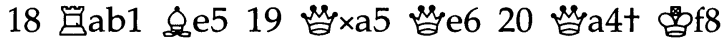
21 \&h6t \&g7 22 침4 f6 23 \&f4 कf7 24

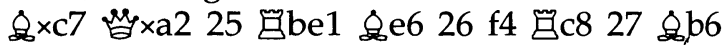

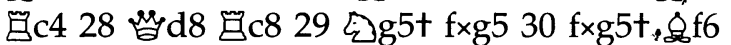

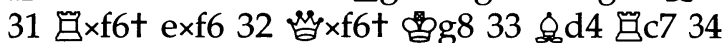

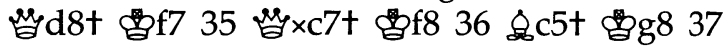
kygly $\mathrm{d} 8+1-0$

\section{Round 4}

\section{Deep Thought - Cray Blitz D01/12 4. Ne4}

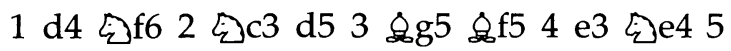

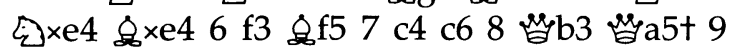

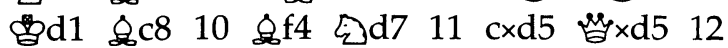

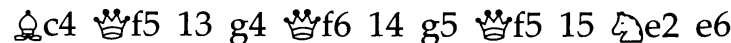

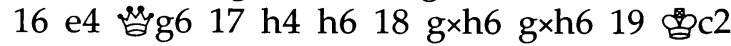

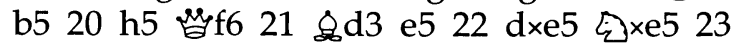

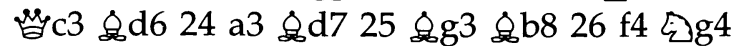

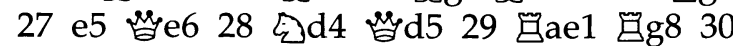

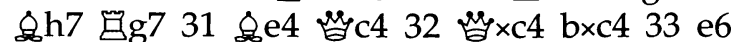

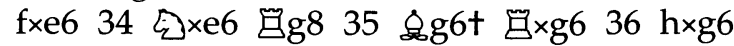

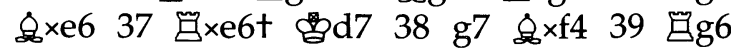

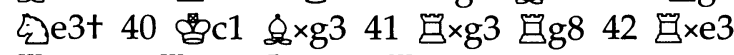

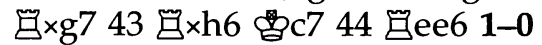

$$
\begin{gathered}
\text { Hitech - Fidelity } \mathbf{X} \\
\text { D02/11 } 5 \text { Nbd2 }
\end{gathered}
$$

$1 \mathrm{~d} 4 \mathrm{~d} 52 \fallingdotseq \mathrm{f} 3 \quad \mathrm{f} 63$ \&f4 \&f5 4 e3 c6 5

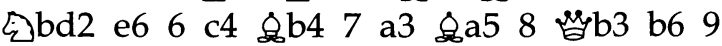

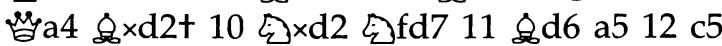

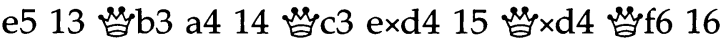

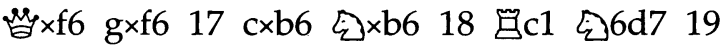

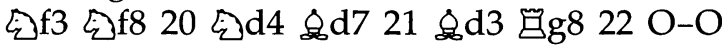
\&3 23 \&3 \&d7 24 e4 §e6 25 ¿f5 d4 26

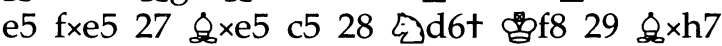

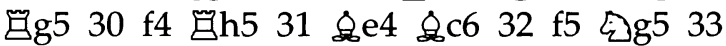

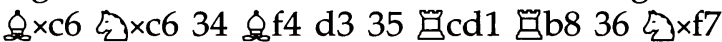

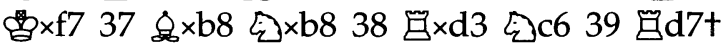

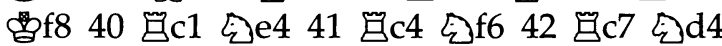

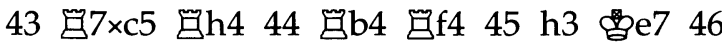

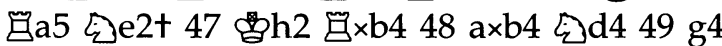

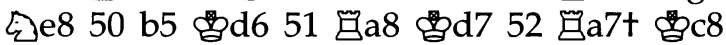

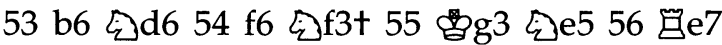

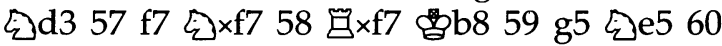
畐7 今c6 1-0

\section{Novag X - Mephisto X D20/13 8. Qf6}

1 d4 d5 2 c4 dxc4 3 e4 e5 4 @f 3 \&b4t 5 \&d2 $\quad \& \times d 2+6 \quad \xi b \times d 2$ exd4 $7 \quad \& \times c 4 \quad \xi c 68$

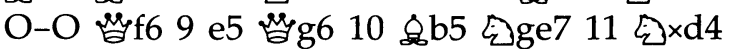

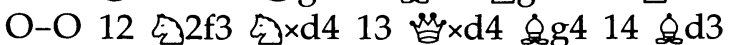

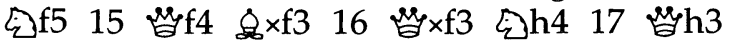

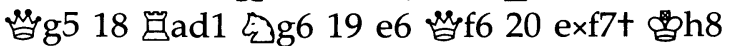

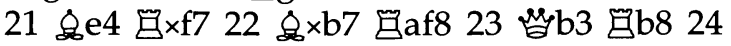

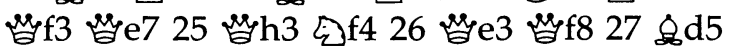

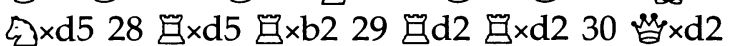

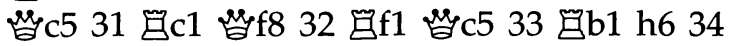
h4 c6 35 gb8t

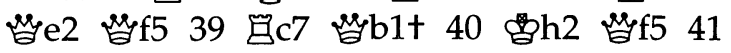

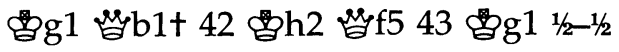

\section{Merlin - Sun Phoenix C02/07 12 Be3}

1 e4 e6 2 d4 4 d5 3 e5 c5 4 d xc5 5 c6 5 亿f3

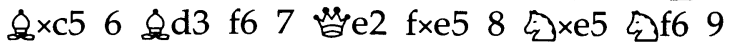
\&f4 O-O 10 O-O ¿e4 11 ¿×c6 b×c6 12 


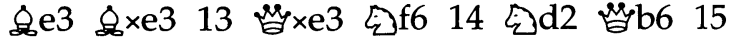

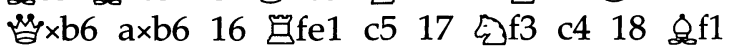

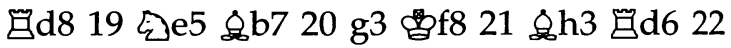

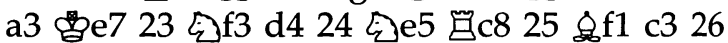

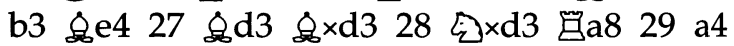

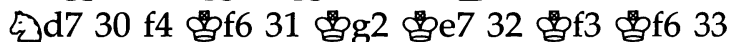

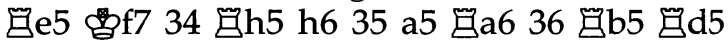
37 Exd5 exd5 38 b4 39 हc1 b×a5 40 昌 $\times$ a5 1-0 time

\section{Bebe - Rebel X A04/01 1. c6}

1 ¿f3 c6 2 \&c3 d5 3 d4 \&f5 4 h3 e6 5 g4

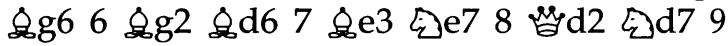

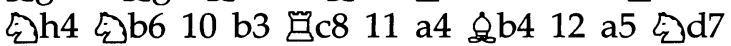

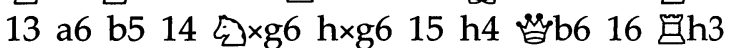
今b8 17 \&4

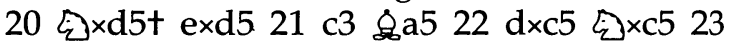

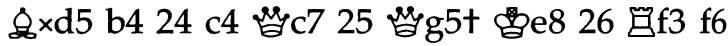

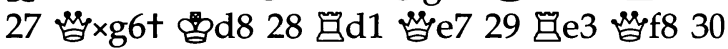

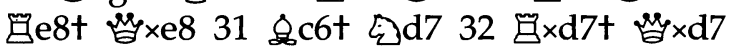

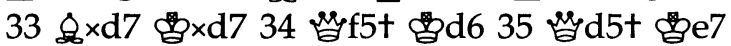

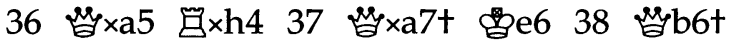

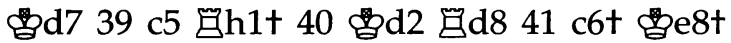

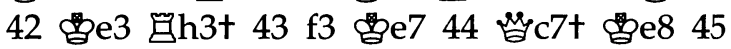
땁. $\times$ 7 1-0

\section{Zarkov - AI Chess} A41/09 5 h3

1 c4 g6 2 d4 \&g7 3 ¿c3 d6 4 ¿f3 乞c6 5 h3

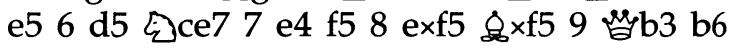

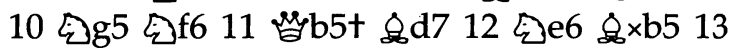

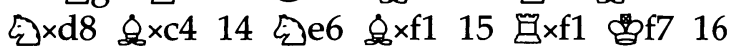

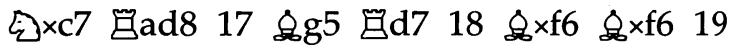

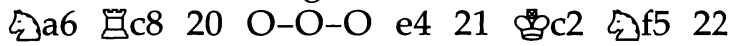

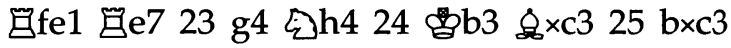

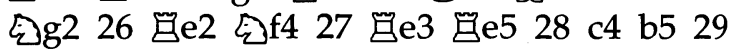
c×b5 $气 \times d 530 \Xi g 3$ e6 31 a4 g5 32 gd2 h6

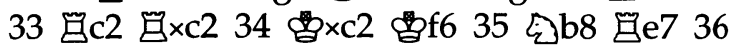

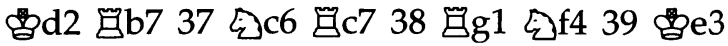

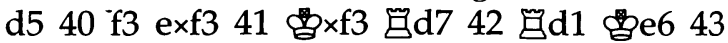

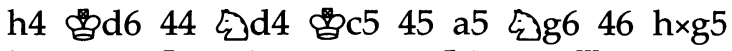

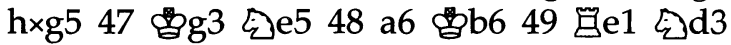

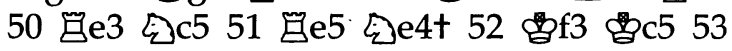

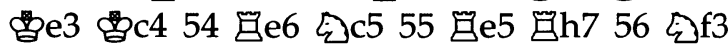

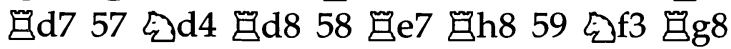

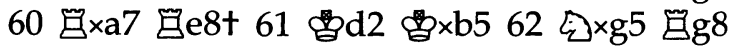

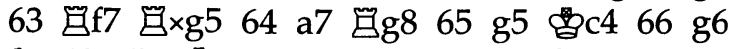

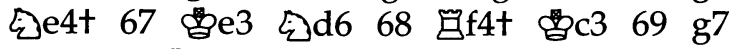

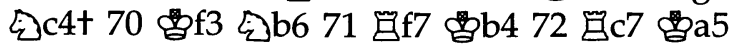

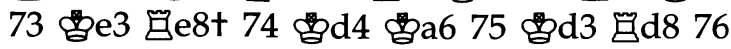
昌f $1 / 2-1 / 2$

$$
\begin{aligned}
& \text { Dappet - Much } \\
& \text { B09/37 7. e5 }
\end{aligned}
$$

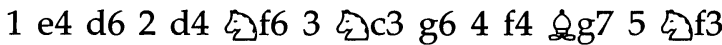
O-O 6 \&d3 7 c6 7 O-O e5 8 fxe5 dxe5 9 d5

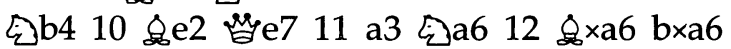

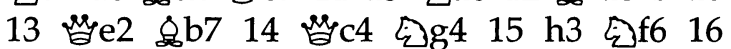

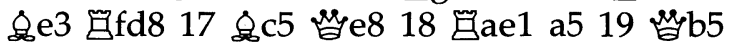

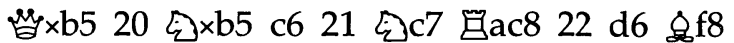

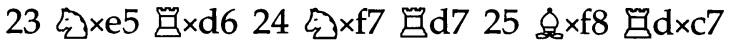

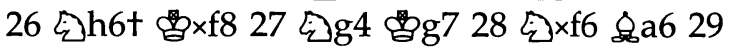

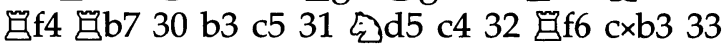

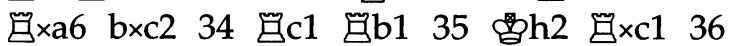

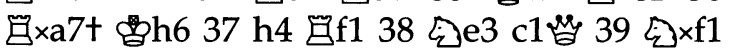

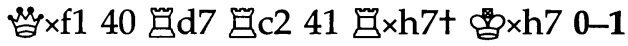

$$
\begin{gathered}
\text { Shess - Quest X } \\
\text { C14/15 9. N:c5 }
\end{gathered}
$$

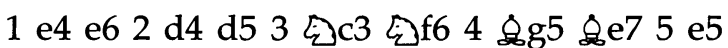

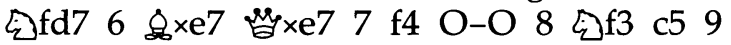

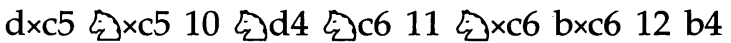
¿d7 13 b5 c5 14 \&e 2 d4 15 ¿e4 \&b7 16

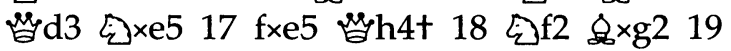

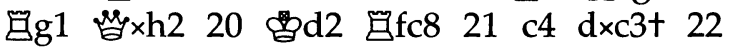

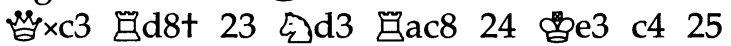

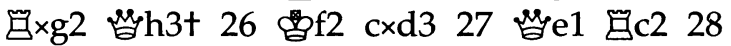

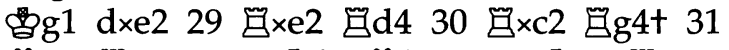

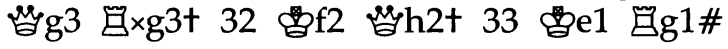
0-1

$$
\begin{gathered}
\text { BP }- \text { Rex } \\
\text { C34/05 8. O-O }
\end{gathered}
$$

1 e4 e5 2 f4 exf4 3 ff3 d6 4 \&c4 h6 5 b3

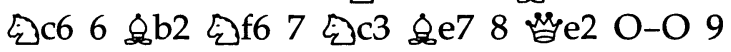

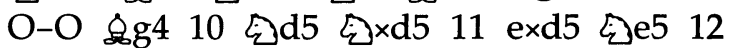

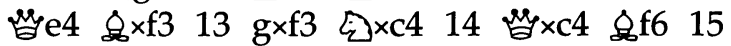

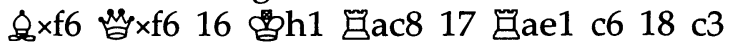

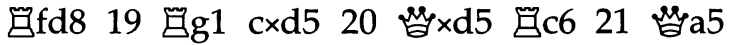

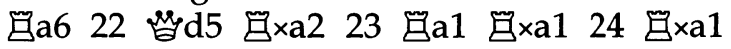

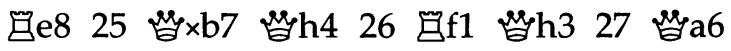
ge5 28 b4 h5 29 쌈4 g6 30 쌉a6 gi 31 쌉c4 a5 32 b5 a4 33 b6 尚c5 34 쌈d4t $\mathrm{f} 635$

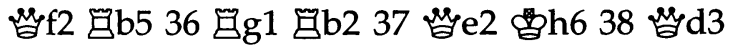
g5 39 Ga1 d5 40 쌈 $\times$ d5 国×b6 41 gुg1 a3 42

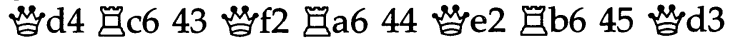

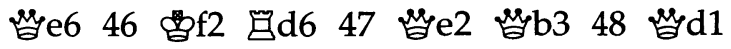

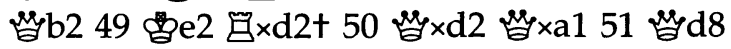

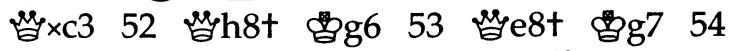

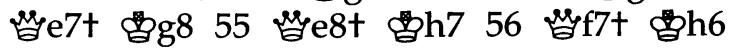
$1 / 2-1 / 2$ 


\section{Y!89 - Waycool B33/31 14 c:d5}

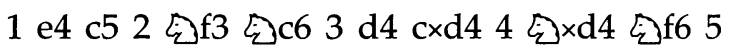
¿c3 e5 6 idb5 d6 7 \&g5 a6 8 \& $\times$ f6 gxf6 9

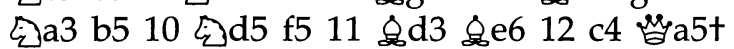
13 off1 \&xd5 14 cxd5 fxe4 15 \&xe4 引e7 16

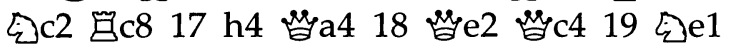

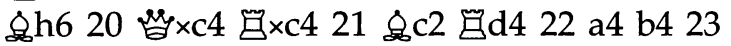

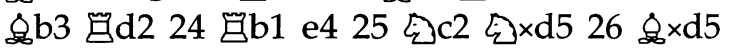

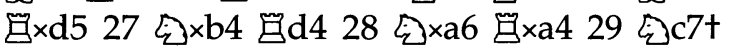

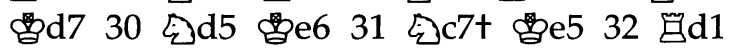

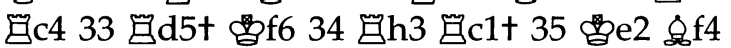

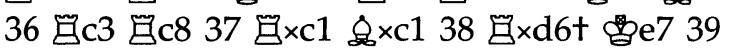

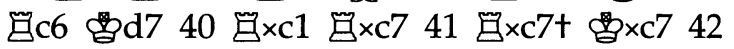

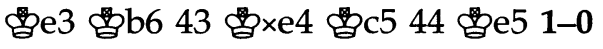

\section{Pandix - Kallisto A06/02 2. Nc6}

$1 乞 f 3$ d5 2 b3 乞c6 3 e3 e5 4 d4 \&g4 5 dxe5

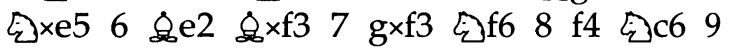

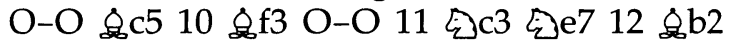
c6 13 ¡a4 \&d6 14 \& $\times$ f6 $\mathrm{g} \times \mathrm{f} 615 \mathrm{c} 4 \mathrm{~d} \times \mathrm{c} 416$

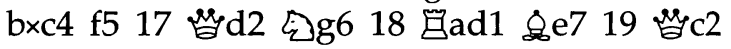

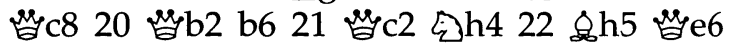

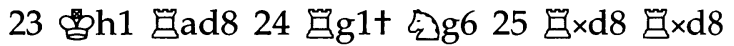

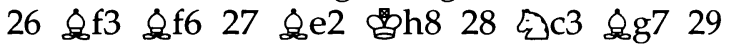

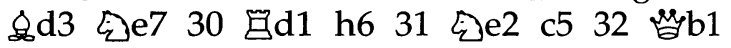

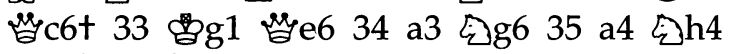

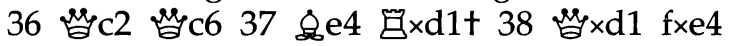

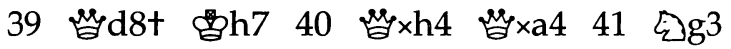

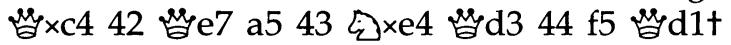

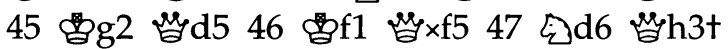

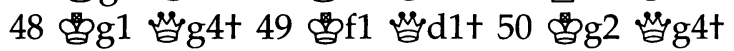

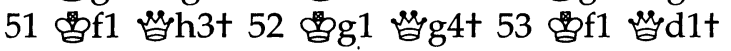
$1 / 2-1 / 2$

\section{Centaur - Moby B50/03 4 e5}

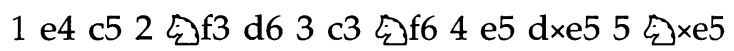

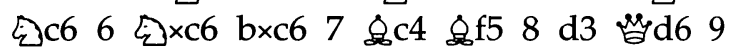

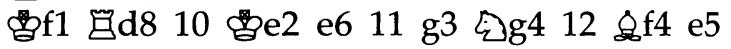
13 \&g5 f6 14 \&e3 $5 \times 2315$ mee3 e4 16 g4

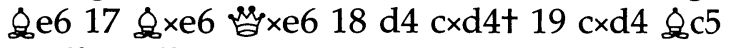

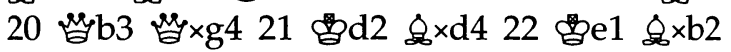

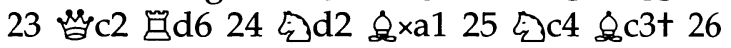

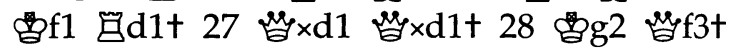
29 gi O-O 30 h3 Eb8 31 ڤe3 छb1+ 0-1

\section{Round 5}

\author{
Hitech - Deep Thought \\ D02/13 4. Nc6
}

$1 \mathrm{~d} 4 \mathrm{~d} 52 \fallingdotseq \mathrm{f} 3 \fallingdotseq \mathrm{f} 63$ \&f4 e6 4 e3 $\leftrightharpoons \mathrm{c} 65$ 乌bd2 \&e7 $6 \mathrm{~h} 3 \mathrm{O}-\mathrm{O} 7$ \&e2 $\mathrm{gh} 5 \mathrm{\& h} 2 \mathrm{~g} 6$

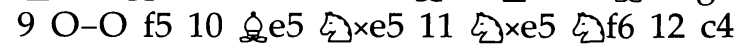
c5 13 @df3 \&d6 14 a3 ․ㅏㅁㄱ 15 घc1 a5 16

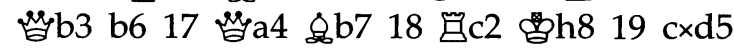

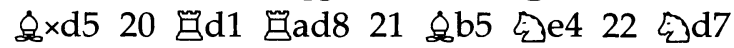

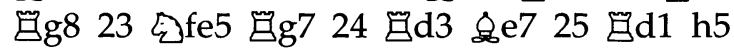

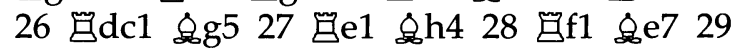
gfc1 g5 $30 \mathrm{f3}$ ¿f6 31 thf1 g4 $32 \mathrm{~h} \times \mathrm{g} 4 \mathrm{~h} \times \mathrm{g} 4$

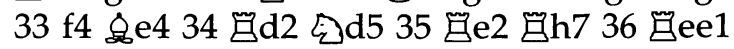

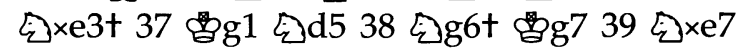

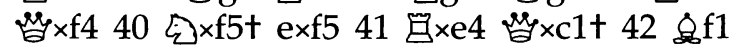

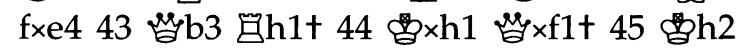

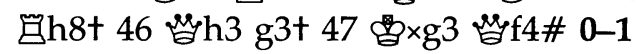

\section{Bebe - Merlin \\ C43/08 9. Be6}

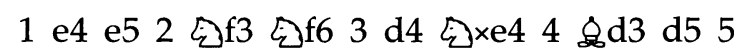

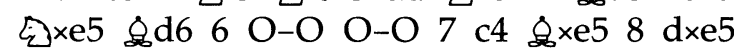

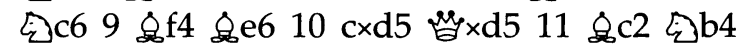

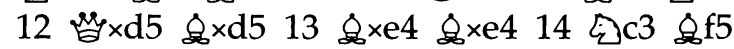
15 b3 $气 d 316$ \&g3 c6 17 f4 gfd8 18 Gad1

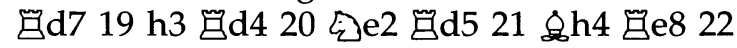

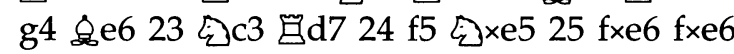

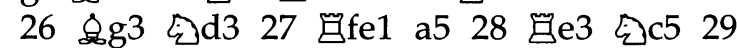

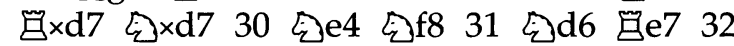

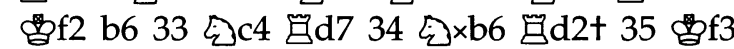

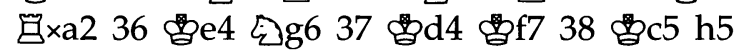

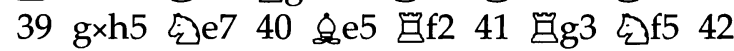

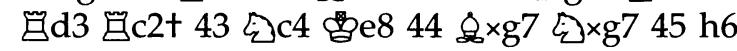

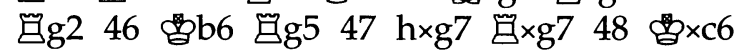

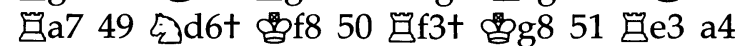

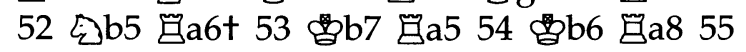

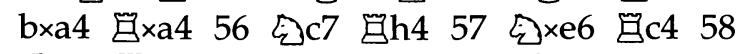

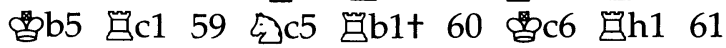

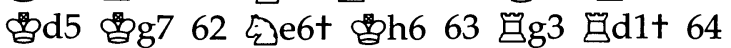

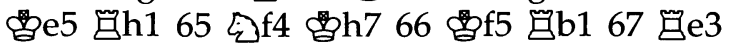

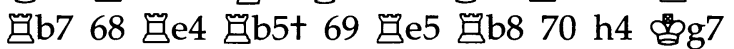

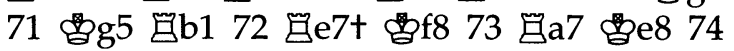

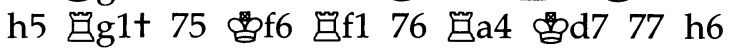

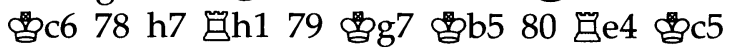
81 ges+1-0

$$
\begin{gathered}
\text { Cray Blitz - Much } \\
\text { B08/08 } 7 \text { Bg5 }
\end{gathered}
$$

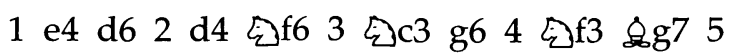

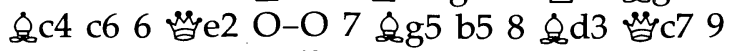

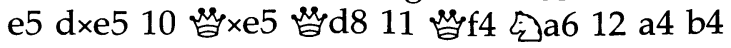




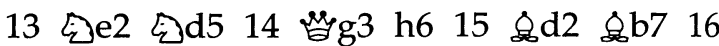

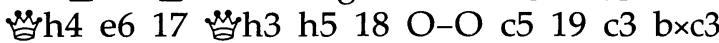

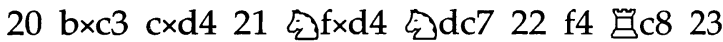

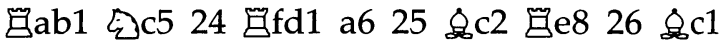

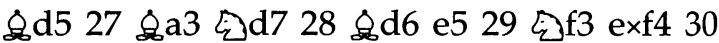

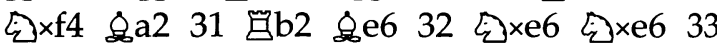

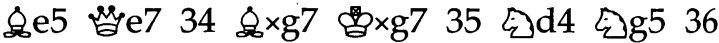

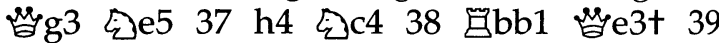

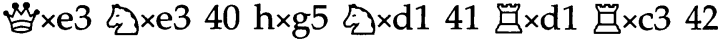

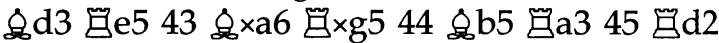

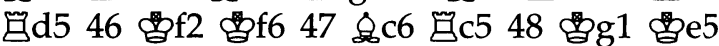

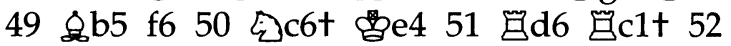
Hh2 53 今d8 ac7 54 \&d3t 55 Ge6t 56 \&e4t 50 \&c4 57 \&c8 58

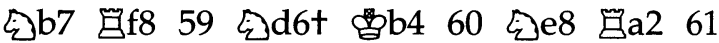

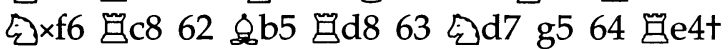

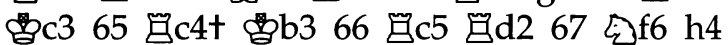

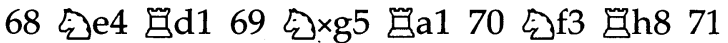

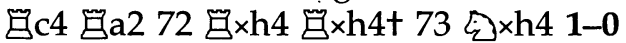

\section{Mephisto X - Zarkov E35/03 6. O-O}

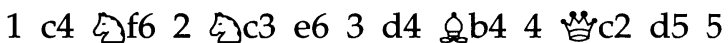
cxd5 exd5 6 \&55 O-O 7 e3 h6 8 \&44 9 c6 9 \&b5 \&d7 10 今ge2 a6 11 \&d3 \&e6 12 O-O

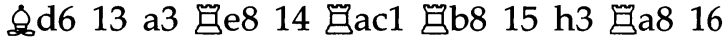

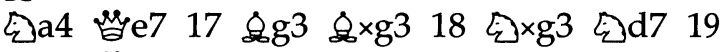

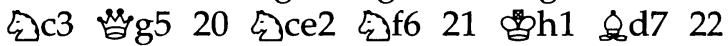

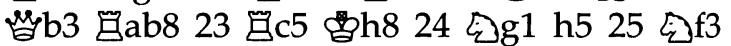

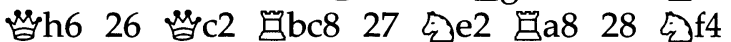

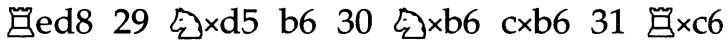

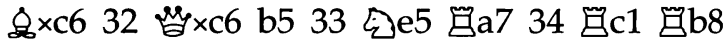

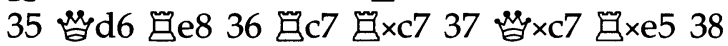
dxe5 $气 g 839$ 쌈xf7 h4 40 쌈e8 g5 41 e6 1-0

\section{Fidelity $X-$ Novag $X$} C78/01 8. Bd7

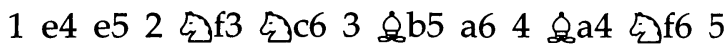
¿c3 \&c5 6 O-O b5 7 \&3 d6 8 d3 \&d7 9

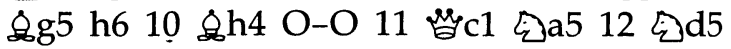

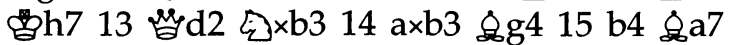

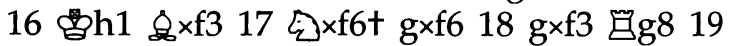

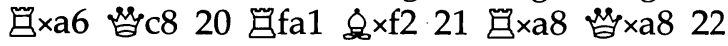

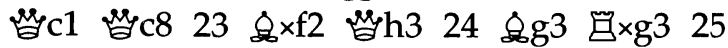

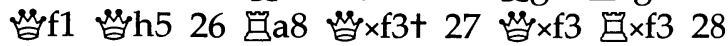

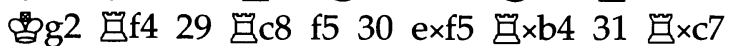

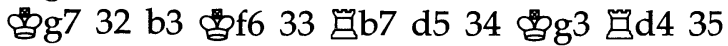

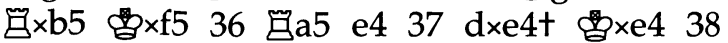

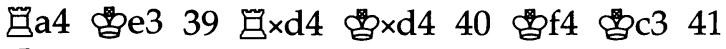

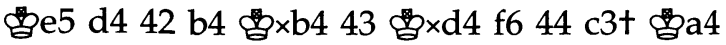

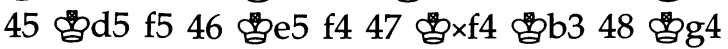

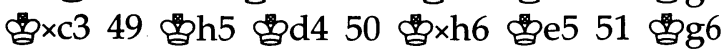

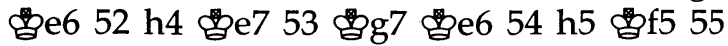

h6 1-0

$$
\begin{gathered}
\text { Sun Phoenix - Y!89 } \\
\text { A29/19 } 16 \text { c:d5 }
\end{gathered}
$$

1 c4 e5 2 ¿n 3 f6 3 g3 \&b4 4 \&g2 O-O 5

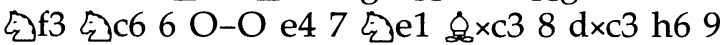

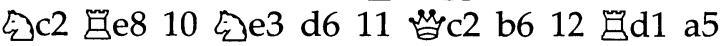

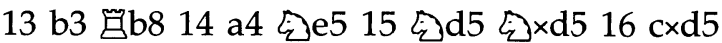

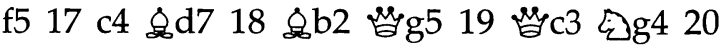

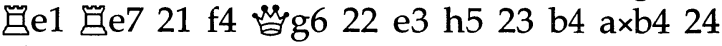

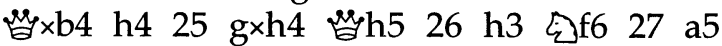

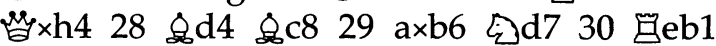

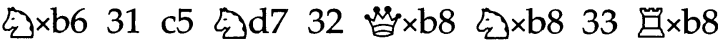

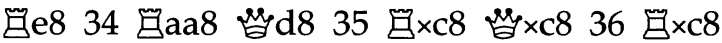

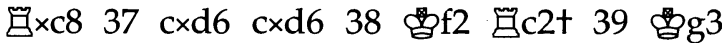

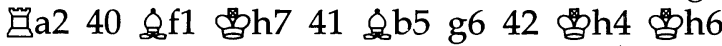

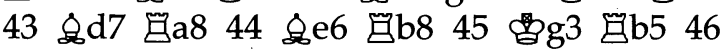

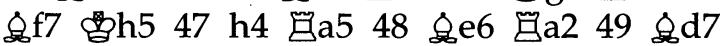

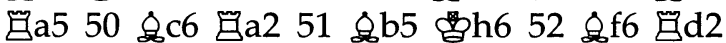

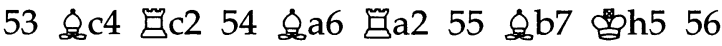

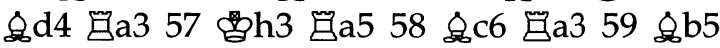

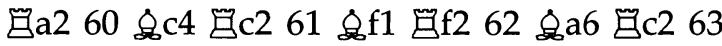

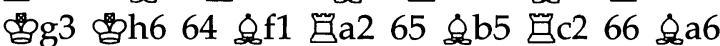

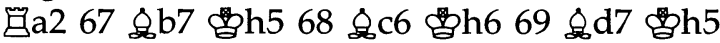

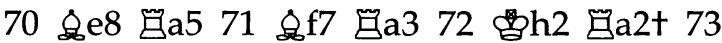

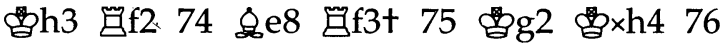
\&b5 Gg3+ 77 fo 2 h $31 / 2-1 / 2$ adjudicated

\section{AI Chess - Rebel X B13/01 4 Nf3}

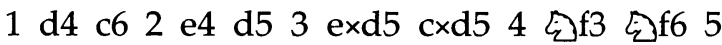

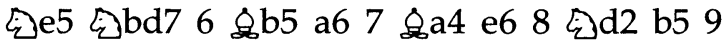
\&b3 \&d6 10 \&df3 \&b7 $11 \quad \& g 5$ kैy 12

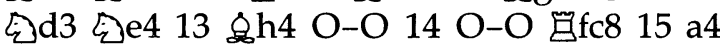

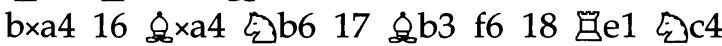

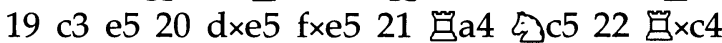

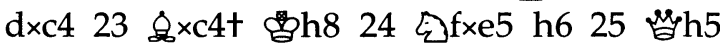

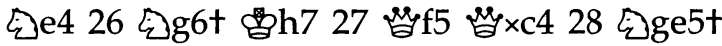

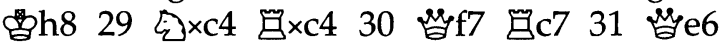

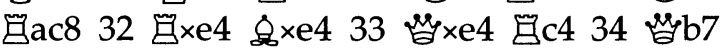

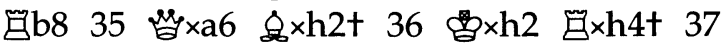

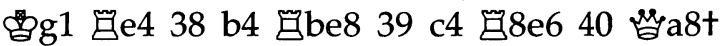
1-0

\section{Quest X - Pandix \\ C32/05 8 Bc4}

1 e4 e5 2 f4 d5 3 exd5 e4 4 d3 $乡$ f6 5 gd2

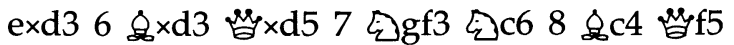

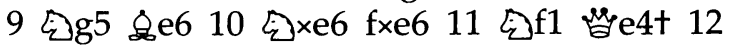

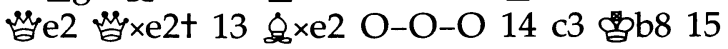

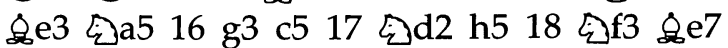

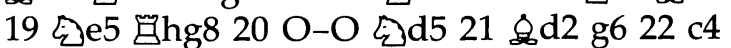

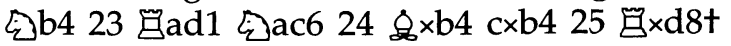




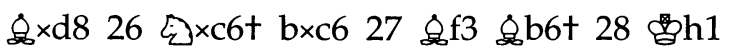
Ha 29 gd1 a5 $30 \mathrm{~h} 4$ \&c5 $31 \mathrm{~b} 3$ \&d6 32

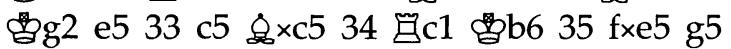

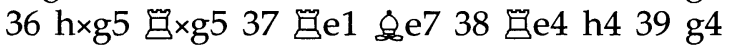

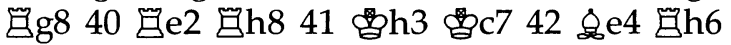

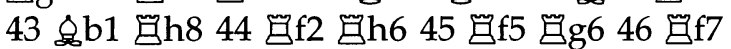

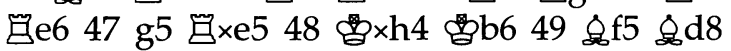

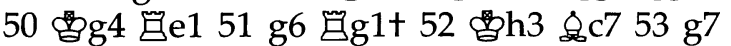

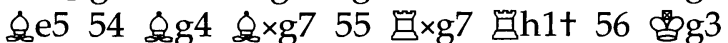

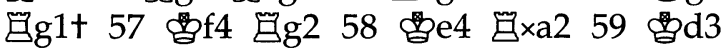

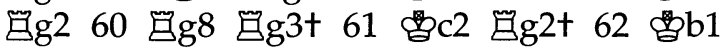

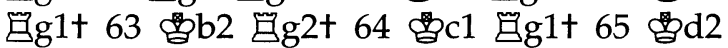

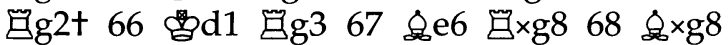
gib5 69 \&c4t 70 \&f 70 \&d4 71 \&

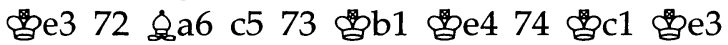

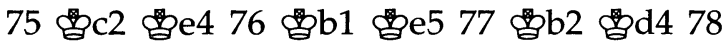

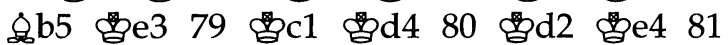

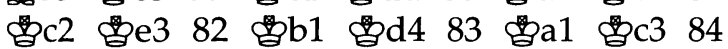

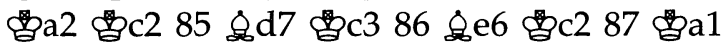

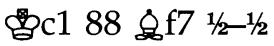

$$
\begin{gathered}
\text { Moby - BP } \\
\text { C42/11 } 10 \text { Ne4 }
\end{gathered}
$$

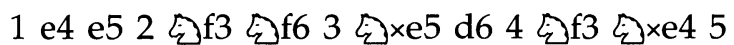

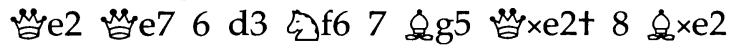

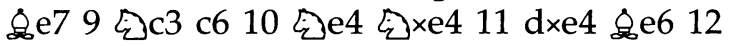

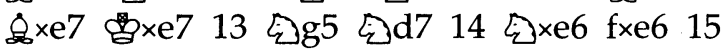

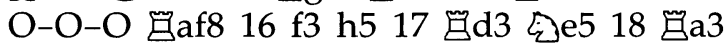

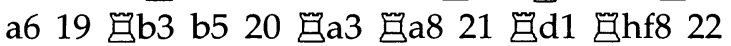

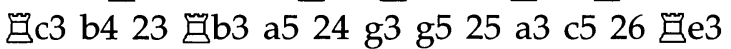

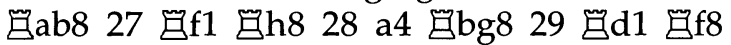

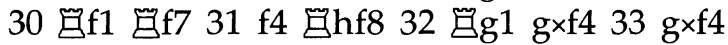

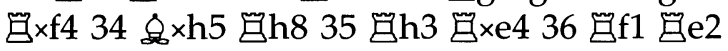

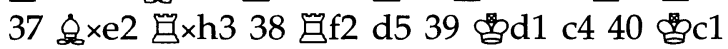

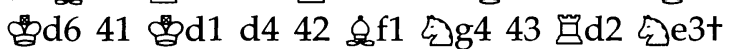
44 ghe1 45 gf2 c3 46 b×c3 b×c3 47 \&d3 e5 $48 \& a 6$ ge4 49 \&d3 $2 \times c 2+0-1$

\section{Rex - Kallisto \\ C68/19 7. Bc5}

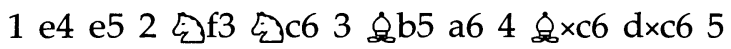

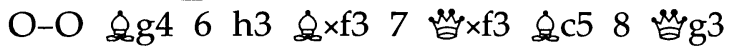

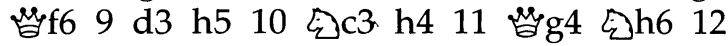

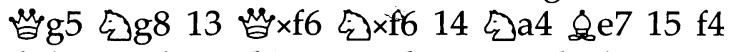
\&d6 16 d4 exd4 17 e5 b5 18 exd6 b×a4 19

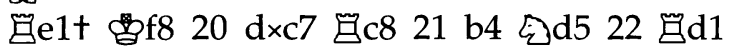

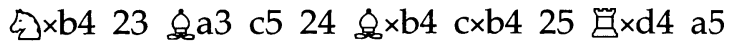
26 Gad1 g6 27 Gd8t 28 G1d7t

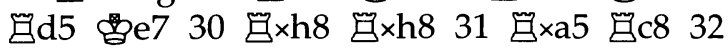

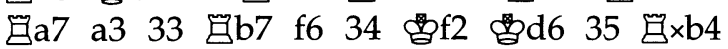

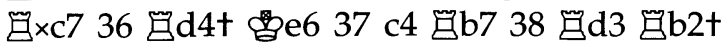

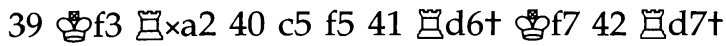

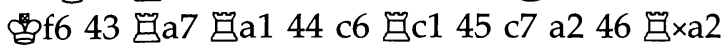

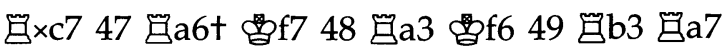
50 日b6t 51 gb2

$$
\begin{gathered}
\text { Dappet - Shess } \\
\text { C54/16 13. Qd6 }
\end{gathered}
$$

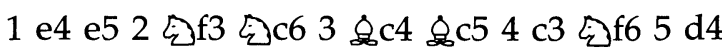
exd4 $6 \mathrm{c} \times \mathrm{d} 4 \& \mathrm{~b} 4+7 \& \mathrm{~d} 2 \& \times \mathrm{d} 2+8 \Leftrightarrow \mathrm{b} \times \mathrm{d} 2 \mathrm{~d} 5$

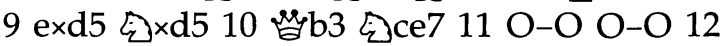

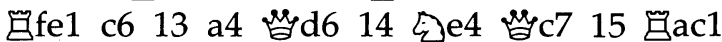

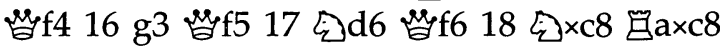

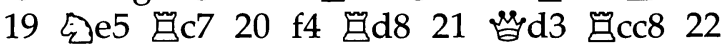

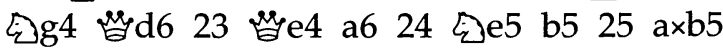

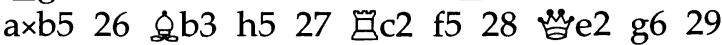

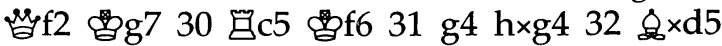

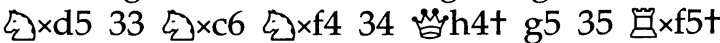

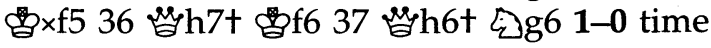

\section{Waycool - Centaur B01/14 5 Bc4}

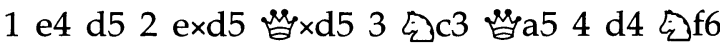

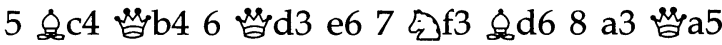

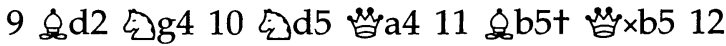

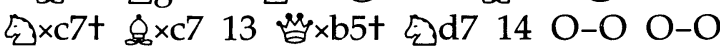
15 कुht \&.h6 g×h6 19 c4 20 Gg1

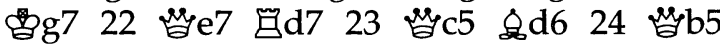

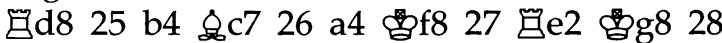

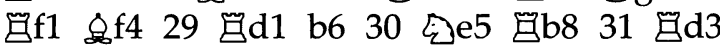

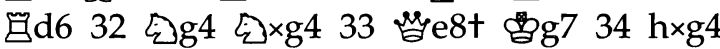

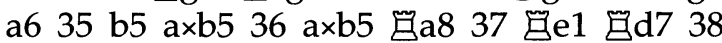

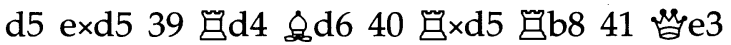
f6 42 日 $\times$ d6 1-0 